\title{
Approximating Nash Social Welfare under Rado Valuations
}

\author{
Jugal Garg* \\ jugal@illinois.edu
}

\author{
Edin Husić ${ }^{\dagger}$ \\ e.husic@lse.ac.uk
}

\author{
László A. Végh ${ }^{\dagger}$ \\ l. vegh@lse.ac.uk
}

\begin{abstract}
We consider the problem of approximating maximum Nash social welfare (NSW) while allocating a set of indivisible items to $n$ agents. The NSW is a popular objective that provides a balanced tradeoff between the often conflicting requirements of fairness and efficiency, defined as the weighted geometric mean of the agents' valuations. For the symmetric additive case of the problem, where agents have the same weight with additive valuations, the first constant-factor approximation algorithm was obtained in 2015. Subsequent work has obtained constant-factor approximation algorithms for the symmetric case under mild generalizations of additive, and $O(n)$-approximation algorithms for subadditive valuations and for the asymmetric case.

In this paper, we make significant progress towards both symmetric and asymmetric NSW problems. We present the first constant-factor approximation algorithm for the symmetric case under Rado valuations. Rado valuations form a general class of valuation functions that arise from maximum cost independent matching problems, including as special cases assignment (OXS) valuations and weighted matroid rank functions. Furthermore, our approach also gives the first constant-factor approximation algorithm for the asymmetric case under Rado valuations, provided that the maximum ratio between the weights is bounded by a constant.
\end{abstract}

\footnotetext{
*University of Illinois at Urbana-Champaign. Supported by NSF Grant CCF-1942321 (CAREER)

${ }^{\dagger}$ Department of Mathematics, London School of Economics and Political Science. This project has received funding from the European Research Council (ERC) under the European Union's Horizon 2020 research and innovation programme (grant agreement no. ScaleOpt-757481).
} 


\section{Contents}

1 Introduction $\quad 1$

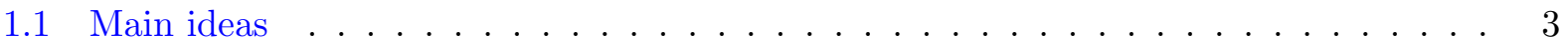

1.2 Further related work . . . . . . . . . . . . . . . . . . 4

2 Preliminaries $\quad \mathbf{5}$

2.1 Valuation functions . . . . . . . . . . . . . . . . . . . 5

2.2 Continuous valuation functions $\ldots \ldots \ldots \ldots \ldots$

2.3 Simple upper bounds . . . . . . . . . . . . . . . . . . . . 9

3 Overview of the approach $\quad 10$

3.1 Phase I: Finding the item set $\mathcal{H} \ldots \ldots \ldots \ldots \ldots$. . . . . . . . . . . . . . .

3.2 Phase II: Reduction to the mixed matching relaxation . . . . . . . . . . . . 11

3.3 Phase III: Approximating the mixed matching relaxation . . . . . . . . . . . . . . 12

3.4 Phase IV: A sparse approximate solution for the mixed matching relaxation . . . . . 13

3.5 Phase V: Rounding the mixed integer solution . . . . . . . . . . . . . . . . 14

4 Phase III: Approximating the mixed matching relaxation 15

4.1 Properties of Eisenberg-Gale program . . . . . . . . . . . . . . . 16

4.2 The approximation guarantee for the mixed matching relaxation $\ldots \ldots$. . . . . 17

5 Phase IV: Obtaining a sparse approximate solution $\quad 19$

5.1 Solving the Eisenberg-Gale relaxation . . . . . . . . . . . . . . . . . . 19

5.2 Sparse solutions to Eisenberg-Gale relaxation . . . . . . . . . . . . . 23

6 Phase V: Rounding the mixed solution 26

6.1 Constructing the new matching . . . . . . . . . . . . . . . . . 29

7 Rado valuations and M-natural-concave functions $\quad 32$

7.1 Rado valuations are M-natural-concave . . . . . . . . . . . . . . . . . . . . 32

7.2 Concave closure of Rado valuations . . . . . . . . . . . . . . . . . . . . 33

7.3 Conjectures on characterizing M-natural-concave functions . . . . . . . . . . . . . 34

8 Connection to spending restricted equilibrium 35

9 Conclusions and future work $\quad 37$ 


\section{Introduction}

In the discrete Nash social welfare (NSW) problem, one needs to allocate a set $\mathcal{G}$ of $m$ indivisible items to a set $\mathcal{A}$ of $n$ agents where each agent $i$ has a valuation function $v_{i}: 2^{\mathcal{G}} \rightarrow \mathbb{R}_{+}$and weight (entitlement) $w_{i}>0$. The goal is to find an allocation maximizing the weighted geometric mean, i.e., the Nash social welfare, of the valuations:

$$
\max \left\{\left(\prod_{i \in \mathcal{A}} v_{i}\left(S_{i}\right)^{w_{i}}\right)^{1 / \sum_{i \in \mathcal{A}} w_{i}}:\left\{S_{i}: i \in \mathcal{A}\right\} \text { forms a partition of } \mathcal{G}\right\} .
$$

We refer to the special case when all agents have equal weight (i.e., $w_{i}=1$ ) as the symmetric NSW problem, and call the general case the asymmetric NSW problem.

The (symmetric) NSW can be seen as a balanced trade-off between two other popular social welfare concepts, the utilitarian social welfare that maximizes the sum of the valuations, and the max-min fairness, also known as the Santa Claus problem, that maximizes the smallest valuation of any agent. A distinctive feature of the NSW problem is invariance under scaling of the valuation functions. That is, unlike the utilitarian social welfare and the max-min fairness, the set of optimal allocations in the NSW problem remains unchanged even if the valuations of the agents are scaled by arbitrary positive constants.

Origins Fair and efficient allocation of resources is a fundamental problem in many disciplines, including computer science, economics, and social choice theory; see, e.g., several excellent books written specifically on this problem $[7,10,11,49,62,63,72]$. The Nash social welfare emerged as an objective that provides a balanced tradeoff between the often conflicting requirements of fairness and efficiency. It was discovered independently in several different contexts: First, as the unique solution to a bargaining game by Nash in 1950 [39, 54]. It also coincides with the notion of competitive equilibrium with equal incomes in economics [68], and as a notion of proportional fairness in networking [40]. The above mentioned works considered the symmetric Nash social welfare problem. The asymmetric objective has also been well-studied since the seventies [34, 38], and has found many applications in different areas, such as bargaining theory [14, 44], water resource allocation [20, 35], and climate agreements [73].

Computational complexity The NSW problem is NP-hard even for two identical agents with additive valuations: the partition problem reduces to the NSW problem [56]. Moreover, the problem is NP-hard to approximate within a factor better than 1.069 for additive valuations [27], and better than 1.5819 for submodular valuations [30]. These results hold already in the symmetric case.

On the positive side, several approximation algorithms were developed for the problem using various techniques. For the symmetric NSW problem with additive valuations, Cole and Gkatzelis [17], in a breakthrough result, designed the first constant-factor approximation algorithm using a spending-restricted market equilibrium. Anari, Oveis Gharan, Saberi, and Singh [1] followed with a constant-factor approximation algorithm built on the theory of real stable polynomials. Barman, Krishnamurthy, and Vaish [8] developed yet another approach based on local search that provides the state-of-the-art approximation factor of 1.45.

These three approaches have been extended to obtain constant-factor approximation algorithms for mild generalizations of additive valuations: budget-additive [28], separable piecewise linear concave (SPLC) [2], and their combination budget-SPLC [15] valuations. All these approaches heavily exploit the symmetry of agents and the characteristics of 'additive-like' valuations, such as the notion of a maximum bang-per-buck (MBB) items, which make them hard to extend to significantly more general settings. 
Beyond 'additive-like' valuations or the asymmetric NSW problem no constant-factor approximation algorithms are known. Here, the state-of-the-art are $O(n)$-approximation algorithms for the asymmetric Nash problem under subadditive valuations $[9,16,30]$. However, no better than $O(n)$ approximation has been achieved even for special cases such as OXS valuations, or only two types of agents with weights 1 or 2 under additive valuations. Therefore, $O(n)$ remained the best approximation factor for the symmetric NSW problem beyond 'additive-like' valuations or for the asymmetric NSW problem.

Independently, in a very recent development, Li and Vondrák [47] gave a $\frac{e^{3}}{(e-1)^{2}}$-approximation of the optimum NSW value for a broad class of submodular valuation functions, including the same class of Rado valuations we study, as well as the cone generated by Rado valuations. A notable example in this cone are coverage functions. The paper extends the real stable polynomial approach used by Anari, Oveis Gharan, Saberi, and Singh [1], and shows that the corresponding convex relaxation has constant integrality gap. However, the randomized rounding technique can find a constant factor approximate solution with exponentially small probability only. Therefore, this does not yield a polynomial-time algorithm for finding a near-optimal allocation. Moreover, the results only apply for symmetric NSW.

Our contributions We make significant progress towards both symmetric and asymmetric NSW problems. Firstly, we obtain a constant-factor approximation for a broad class of submodular valuations we call Rado valuations. ${ }^{1}$ This is a common generalization of OXS valuations and weighted matroid functions. A Rado valuation of an agent $i \in \mathcal{A}$ is specified by a bipartite graph $\left(\mathcal{G}, V_{i} ; E_{i}\right)$, edge $\operatorname{costs} c_{i}: E_{i} \rightarrow \mathbb{R}_{+}$and a matroid $\mathcal{M}_{i}=\left(V_{i}, \mathcal{I}_{i}\right)$. The value $v_{i}(S)$ of a subset of items $S \subseteq \mathcal{G}$ is given as the maximum cost of a matching between nodes in $S$ and nodes in $V_{i}$ such that the endpoints in $V_{i}$ form an independent set in the matroid $\mathcal{M}_{i}$. Relation between popular classes of valuations functions follows $[45,57]$ :

$$
\text { Additive } \subsetneq \text { SPLC } \subsetneq \text { Weighted Matroid Rank } \subsetneq \text { Rado } \subsetneq \text { GS } \subsetneq \text { Submodular } \subsetneq \text { Subadditive }
$$

Theorem 1.1. There exists a polynomial-time $256 e^{3 / e} \approx 772$-approximation algorithm for the symmetric Nash social welfare problem under Rado valuations.

Rado valuations form a subclass of gross substitutes (GS) valuations. In fact, it was conjectured by Frank in 2003 that every GS valuation arises as a Rado valuation, see Section 2.2. In Section 7.3 we give a counterexample and formulate a refinement of this conjecture.

Secondly, we obtain a constant-factor approximation for the asymmetric NSW problem under Rado valuations, provided that the maximum ratio between the weights is bounded by a constant. Assume the weights $w_{i}$ of the agents fall in the interval $[1, \gamma-1]$ for some $\gamma \geq 2$.

Theorem 1.2 (Main). There exists a polynomial-time $256 \gamma^{3}$-approximation algorithm for the Nash social welfare problem with Rado valuation functions. For additive valuation functions, there exists a polynomial-time $16 \gamma$-approximation algorithm.

\footnotetext{
${ }^{1}$ We propose the name "Rado valuations" (Definition 2.4) in honor of Richard Rado, who first studied the independent matching problem [61]. As already mentioned, in the context of NSW, the same class has been studied in the recent work of Li and Vondrák [47] as valuations arising via bipartite matching with a matroid constraint. Murota [53] calls Rado valuations by the name independent assignment valuations. While the name "matroid matching valuations" sounds appealing, we note that matroid matching refers to a different concept in combinatorial optimization [48].
} 
We note that even if the weights of the agents are bounded, an $O(1)$-approximation for the symmetric case does not yield an $O(1)$-approximation to the asymmetric case. ${ }^{2}$ Table 1 summarizes the updated best approximation guarantees for the problem under various valuation functions. The result of $\mathrm{Li}$ and Vondrák [47] gives a $\frac{e^{3}}{(e-1)^{2}} \approx 6.8$ approximation of the optimum value (but without providing an allocation) for the cone generated by Rado valuations in the symmetric case.

\begin{tabular}{|c|c|c|}
\hline Valuations & Symmetric & Asymmetric \\
\hline \hline Additive & $1.45[8]$ & $O(\gamma)[$ Theorem 1.2] \\
\hline SPLC & $1.45[15]$ & $O\left(\gamma^{3}\right)[$ Theorem 1.2] \\
\hline Rado & $O(1)[$ Theorem 1.1$]$ & $O\left(\gamma^{3}\right)[$ Theorem 1.2] \\
\hline Subadditive & $O(n)[9,16]$ & $O(n)[9,16]$ \\
\hline
\end{tabular}

Table 1: Summary of the best approximation algorithms for the NSW problem. Definitions of valuations functions are deferred to Section 2.1.

\section{$1.1 \quad$ Main ideas}

Our approach is based on a mixed-integer programming relaxation, using a careful combination of convex programming relaxations and combinatorial arguments.

The NSW problem is given with discrete valuation functions $v_{i}: 2^{\mathcal{G}} \rightarrow \mathbb{R}_{+}$. In order to apply convex programming techniques, we first need to obtain a convex programming relaxation; already this turns out to be a nontrivial task. As explained in Section 2.2, gross substitute valuations are the subclass of discrete valuations where a concave extension can be naturally defined.

Already for additive valuations, the natural relaxation of the NSW problem has unbounded integrality gap [17]. In order to formulate a mixed integer program, we identify a set $\mathcal{H}$ of $n$ items, and require that all these items must be integrally allocated. We do not know if this relaxation can be solved in polynomial time: we only provide an approximate solution to a further relaxation.

For the set $\mathcal{H}$, we aim to identify the set of the 'most important' items. We find the allocation maximizing the NSW value assuming each agent can obtain just a single item, and select $\mathcal{H}$ as the set of the items chosen in this allocation. This can be efficiently solved as a maximum weight matching problem. The algorithm in [30] also starts with such a matching. One cannot commit to assigning these items to the agents, as it may result in an arbitrarily bad outcome; the approach in [30] is an intricate combinatorial scheme with iterated matchings and reallocations to obtain an $O(n \log n)$ approximation for submodular valuations. Our result implies that the mixed integer relaxation that requires $\mathcal{H}$ to be integrally allocated has a constant integrality gap, in contrast to the standard continuous relaxation. As a possible explanation why this may happen, we make a connection to the approach of Cole and Gkatzelis [17] in Section 8, showing that all 'expensive' items in the spending restricted equilibrium will be included in $\mathcal{H}$.

We give a detailed exposition of the overall approach and formulate the main lemmas in Section 3, split into five phases. Here, we only give a high-level overview. Phase $\mathbf{I}$ selects $\mathcal{H}$ as above. Phase II approximates the mixed relaxation by another mixed integer program (Mixed+matching) that assigns items $\mathcal{G} \backslash \mathcal{H}$ fractionally to the agents, and at most one item from $\mathcal{H}$ to each agent. This is not a relaxation of the original problem anymore, as an optimal solution may allocate multiple

\footnotetext{
${ }^{2}$ To illustrate this point, consider two items and two agents with weights $w_{1}=2, w_{2}=1$ and additive valuations $v_{1}(\{1\})=M, v_{1}(\{2\})=1, v_{2}(\{1\})=M+1, v_{2}(\{2\})=1$, where $M$ is an arbitrarily large number. The unique optimal solution to the symmetric case (by setting $w_{1}^{\prime}=w_{2}^{\prime}=1$ ) is allocating good 2 to agent 1 and good 1 to agent 2. However, this returns an NSW value $(M+1)^{1 / 3}$ for the original weights. This can be worse by an arbitrary factor than the value $M^{2 / 3}$ obtainable by assigning good 1 to agent 1 and good 2 to agent 2 .
} 
items from $\mathcal{H}$ to the same agent. However, (Mixed+matching) approximates the original mixed within a factor $\gamma$. We note that this is the only part of our reductions that depends on the bound $\gamma$.

Solving (Mixed+matching) still does not turn out to be easy. In Phase III, we find a 2approximate solution by first solving the restriction to $\mathcal{G} \backslash \mathcal{H}$ - a convex program - then optimally assigning the items in $\mathcal{H}$ subject to this fractional allocation.

All reductions thus far work for general subadditive valuations, assuming they are given with a suitable concave extension. In Phase IV we exploit combinatorial properties of the concave extension of Rado valuations to obtain a sparse solution. We first show that the restriction of (Mixed+matching) to $\mathcal{G} \backslash \mathcal{H}$ has a basic optimal solution with at most $|\mathcal{A}|+2|\mathcal{G} \backslash \mathcal{H}|$ nonzero variables. We note that this yields an interesting new rational convex program [69], the first nonlinear example we are aware of with an exponential number of constraints, given by a separation oracle. We then further sparsify the solution to at most $2|\mathcal{A}|+|\mathcal{G} \backslash \mathcal{H}|$ non-zero variables, at the expense of losing at most half of the objective value.

At this point, we have a mixed integer solution that is not too far from an integral one. Namely, $\mathcal{H}$ is already allocated integrally and $\mathcal{G} \backslash \mathcal{H}$ is allocated to agents fractionally but with at most $2|\mathcal{A}|+|\mathcal{G} \backslash \mathcal{H}|$ non-zero variables. Thus, it suffices to fix a suitable subset of $2|\mathcal{A}|$ fractional variables to zero of the non-zero to obtain a feasible solution, and round the rest of the variables to 1 . However, this may not be viable for any subset.

In the final Phase $\mathbf{V}$, we make use of the initial choice of $\mathcal{H}$ as the set of items allocated in the best allocation with one item per agent. Using this property, we carefully recombine the matching in the mixed assignment and the initial allocation of the items in $\mathcal{H}$ by swapping around alternating cycles. This enables the final rounding step to obtain an integer allocation.

We note that Phase IV and Phase $\mathbf{V}$ are the most involved in our approach.

\subsection{Further related work}

We briefly mention further results on Nash social welfare, utilitarian social welfare and max-min welfare.

Nash social welfare NSW has turned out to be the focal point in fair division. Caragiannis, Kurokawa, Moulin, Procaccia, Shah, and Wang [13] call the optimal NSW solution 'unreasonably' fair and efficient. The same paper introduces an algorithm for finding optimum NSW allocation, which is deployed on the website spliddit.org and used for fair allocation of indivisible goods [31]. Approximation algorithms for the NSW also preserve many nice fairness properties, as shown in $[12,16,26]$.

Utilitarian social welfare In this setting, the goal is to find a partition of the items that maximizes the sum of agents' valuations. This problem is straightforward for additive valuations. For gross substitutes valuations (see Definition 2.1), the optimal partition corresponds to a Walrasian equilibrium: there exists a price vector such that each agent receives an optimal bundle at these prices. Such an allocation can be efficiently computed [33, 41]. Gül and Stachetti [33] also showed that the converse is essentially true: if a class $\mathcal{C}$ of valuation functions contains all unit demand valuations, and there exists a Walrasian equilibrium for an arbitrary choice of valuation functions from $\mathcal{C}$, then $\mathcal{C}$ must be a subset of gross substitutes valuations.

For submodular valuations there is an $\frac{e}{e-1} \approx 1.5819$-approximation algorithm by Vondrák [71] and this is the best possible [43]. Feige [24] gave a 2-approximation algorithm for the social welfare problem under subadditive valuations assuming access to particular demand queries.

Max-min welfare In this problem the objective is to maximize the minimum valuation of any agent. This NP-hard problem can be seen as an absolute fairness problem and it has been ap- 
propriately named the Santa Claus problem [6]. It is a significant open problem to obtain a constant-factor approximation for additive valuations: such algorithms are known only for restricted subclasses of additive valuations, see Annamalai, Kalaitzis, and Svensson [3], and Davies, Rothvoß, and Zhang [19]. For additive (resp. submodular) valuations the best approximation factor is $O\left(\sqrt[3]{n} \log ^{3} n\right)$ by Asadpour and Saberi [4] (resp. $O(n)$ by Khot and Ponnuswami [42]).

Organization of the paper In Section 2 we formally define all the notation and concepts. Here, we also explain the significance of the gross substitutes and Rado valuations for the problem and our approach. Missing proofs from Section 2, as well as a detailed discussion of Rado valuations are presented in Section 7. In Section 3 we give a rigorous overview of the algorithm together with main lemmas proof ideas. Sections 4-6 contain more detailed arguments for the various phases. Section 8 compares our approach with the spending restricted equilibria in [17]. Concluding remarks are given in Section 9.

\section{Preliminaries}

Throughout, we let $\mathcal{G}$ denote a finite set of $m$ indivisible items (goods), and $\mathcal{A}$ a set of $n$ agents. Each of the agents $i \in \mathcal{A}$ are equipped with a valuation function $v_{i}: 2^{\mathcal{G}} \rightarrow \mathbb{R}_{+}$. We use the shorthand notation $v_{i j}=v_{i}(\{j\})$ to denote the valuation of agent $i$ for the whole item $j$.

Given a subset $S \subseteq \mathcal{G}$ we denote with $\chi_{S}$ the characteristic vector of $S$. For $k \in \mathbb{N}$, we let $[k]=\{1,2, \ldots, k\}$. A bipartite graph $(U, V ; E)$ has node set $U \cup V$ and an undirected edge set $E \subseteq U \times V$. For an edge subset $F \subseteq E$, we let $\delta_{U}(F)$ and $\delta_{V}(F)$ denote the set of endpoints of $F$ in $U$ and in $V$, respectively. A matching from $U$ to $V$ is represented as a mapping $\sigma: U \rightarrow V \cup\{\emptyset\}$ such that for each $i \in U$ with $\sigma(i) \neq \emptyset$, we have $(i, \sigma(i)) \in E$, and if $\sigma(i) \neq \emptyset$, then $\sigma(i) \neq \sigma(j)$ for $j \neq i$.

A matroid on a finite ground set $V$ is given as $\mathcal{M}=(V, \mathcal{I})$, where $\mathcal{I} \subseteq 2^{V}$ is a nonempty collection of independent sets. This collection is required to satisfy the independence axioms:

(I1) Monotonicity: if $X \in \mathcal{I}$ then $Y \in \mathcal{I}$ for all $Y \subseteq X$, and

(I2) Exchange property: if $X, Y \in \mathcal{I},|X|<|Y|$, then there exists a $y \in Y \backslash X$ such that $X \cup\{y\} \in \mathcal{I}$.

The rank function $r_{\mathcal{M}}: 2^{V} \rightarrow \mathbb{Z}_{+}$associated with the matroid $\mathcal{M}$ is defined with $r_{\mathcal{M}}(X)$ denoting the size of the largest independent subset of $X \subseteq V$. A fundamental property implied by (I2) is that every maximal independent set in $X$ has size $r_{\mathcal{M}}(X)$. The value $r_{\mathcal{M}}(V)$ is called the rank of the matroid, and the maximal independent sets are called bases. A set $X \subseteq V$ is in $\mathcal{I}$ if and only if $r(X)=|X|$. We refer the reader to [65, Part IV] for matroids and their role in optimization.

\subsection{Valuation functions}

By a valuation function, we mean a function $v: 2^{\mathcal{G}} \rightarrow \mathbb{R}_{+}$with $v(\emptyset)=0$. Let us start with two simple examples of valuations. The function $v$ is an additive valuation if $v(S)=\sum_{j \in S} v_{j}$, and a unit demand valuation if $v(S)=\max _{j \in S} v_{j}$ where $v_{j} \in \mathbb{R}_{+}$represents the value of item $j \in \mathcal{G}$.

We now define some basic properties. A function $v: 2^{\mathcal{G}} \rightarrow \mathbb{R}_{+}$is monotone if $v(X) \leq v(Y)$ for any $X \subseteq Y \subseteq \mathcal{G}$, subadditive if

$$
v(X)+v(Y) \geq v(X \cup Y) \quad \forall X, Y \subseteq \mathcal{G},
$$

and submodular if

$$
v(X)+v(Y) \geq v(X \cap Y)+v(X \cup Y) \quad \forall X, Y \subseteq \mathcal{G} .
$$

Additive valuations and unit demand valuations satisfy all the above properties. Another basic example of submodular functions is the rank function $r_{\mathcal{M}}$ of a matroid $\mathcal{M}=(V, \mathcal{I})$. In fact, every 
integer valued monotone submodular set function on $V$ with $v(X) \leq|X|$ arises as the rank function of a matroid. Given a weighting $g \in \mathbb{R}_{+}^{V}$, the weighted rank function $r_{g}(X)$ is the maximum $g$-weight of a maximal independent set in $X$; this function is also submodular.

Gross substitute valuations For a price vector $p \in \mathbb{R}^{\mathcal{G}}$ and a subset $S \subseteq \mathcal{G}$, we let $p(S)=$ $\sum_{j \in S} p_{j}$. For a valuation function $v: 2^{\mathcal{G}} \rightarrow \mathbb{R}_{+}$, the utility obtainable at prices $p$ from a set $S \subseteq \mathcal{G}$ is $v(S)-p(S)$. The set of optimal bundles at prices $p$ is called demand correspondence and is defined as the set of bundles maximizing the utility, i.e.,

$$
D(v, p):=\underset{S \subseteq \mathcal{G}}{\arg \max } v(S)-p(S) .
$$

An important class of valuation functions is gross substitutes valuations, defined by Kelso and Crawford in 1982 [41]:

Definition 2.1. The valuation function $v: 2^{\mathcal{G}} \rightarrow \mathbb{R}_{+}$is a gross substitutes (GS) valuation if for any $p, p^{\prime} \in \mathbb{R}^{\mathcal{G}}$ such that $p^{\prime} \geq p$ and any $S \in D(v, p)$, there exists an $S^{\prime} \in D\left(v, p^{\prime}\right)$ such that $S \cap\left\{j: p_{j}=p_{j}^{\prime}\right\} \subseteq S^{\prime}$

That is, if we have an optimal bundle at prices $p$ and increase some of the prices, then there will be an optimal bundle that contains all items whose price remained unchanged. For a comprehensive survey on GS valuations, we refer the reader to the survey by Paes Leme [60].

Gül and Stachetti [33] showed that every gross substitutes valuation is submodular. It turns out that gross substitute functions are intimately connected to discrete convex analysis, a general theory arising at the intersection of convex analysis and submodularity.

Murota's book [50] gives a comprehensive treatment of this field. A central concavity concept on the integer lattice is that of $M^{\natural}$-concave functions. The definition specialized for valuation functions (corresponding to the sublattice $\{-\infty, 0\}^{\mathcal{G}}$ ) is as follows.

Definition 2.2. The function $v: 2^{\mathcal{G}} \rightarrow \mathbb{R}_{+}$is an $M^{\natural}$-concave if for any $X, Y \subseteq \mathcal{G}$ and $x \in X \backslash Y$,

$$
v(X)+v(Y) \leq \max _{Z \subseteq Y \backslash X,|Z| \leq 1} v((X \backslash\{x\}) \cup Z)+v((Y \backslash Z) \cup\{x\})
$$

That is, for any $x \in X \backslash Y$, the sum $v(X)+v(Y)$ is either non-decreasing if we move $x$ from $X$ to $Y$, or the sum is non-decreasing by swapping $x$ for some $y \in Y \backslash X$. As established by Fujishige and Yang [25], these two concepts are equivalent:

Theorem 2.3 ([25]). The valuation function $v: 2^{\mathcal{G}} \rightarrow \mathbb{R}_{+}$is a gross substitutes valuation if and only if it is $M^{\natural}$-concave.

This connection has enabled a fruitful interaction between the areas of mechanism design and discrete convexity, see e.g. [53, 60].

Rado valuations The key class of valuation functions for this paper will be Rado valuation functions, or Rado valuations. We provide examples and an intuitive interpretation of these valuations after the definition.

Definition 2.4. Assume we are given a bipartite graph $(\mathcal{G}, V ; E)$ with a cost function $c: E \rightarrow \mathbb{R}_{+}$ on the edges, and a matroid $\mathcal{M}=(V, \mathcal{I})$. For a subset of items $S \subseteq \mathcal{G}$, the Rado valuation function $v(S)$ is defined as the maximum cost of a matching $M$ in $(\mathcal{G}, V ; E)$ such that $\delta_{\mathcal{G}}(M) \subseteq S$ and $\delta_{V}(M) \in \mathcal{I}$, i.e.,

$$
v(S):=\max \left\{\sum_{e \in M} c(e): M \text { is a matching, } \delta_{\mathcal{G}}(M) \subseteq S, \delta_{V}(M) \in \mathcal{I}\right\} .
$$


Let us consider the special case where the matroid $\mathcal{M}$ is the free matroid on $V$, i.e., $\mathcal{I}=2^{V}$. In this case, the matroid constraints $\delta_{V}(M) \in \mathcal{I}$ are void. The value of a set $S$ it then the maximum cost matching in the bipartite subgraph induced by $S \cup V$. Such valuations are called assignment valuations by Shapley [66], and OXS valuations by Lehmann, Lehmann, and Nisan [45].

Shapley [66] gives a nice interpretation of assignment valuations. Assume that each agent is a company. Furthermore, assume that the items $\mathcal{G}$ are workers and $V$ is the set of jobs within a particular company. The edge set represents the possibilities (willingness) of assigning workers to jobs, and the cost $c_{j k}$ is value the company gets by assigning worker $j$ to job $k$. By the definition of assignment valuations, the value of a subset $S \subseteq \mathcal{G}$ of workers for the company is the maximum possible value the company gets by assigning workers $S$ to jobs $V$.

The same interpretation extends to Rado valuations with the additional possibility that the occupied set of jobs must be an independent set in matroid $\mathcal{M}$. For example, the company may partition the set of all jobs $V$ into certain types, and require that at most one job of each type to be assigned - a partition matroid constraint.

As another example of Rado valuations, consider the case where $V$ is a copy of the set of items $\mathcal{G}$, with each $j \in \mathcal{G}$ having a corresponding $j^{\prime} \in V$, and let $E=\left\{\left(j, j^{\prime}\right): j \in \mathcal{G}\right\}$. Let $g: \mathcal{G} \rightarrow \mathbb{R}$, and $c_{j j^{\prime}}=g_{j}$ for all $j \in \mathcal{G}$, and let $r$ be rank function of $\mathcal{M}$. In this case the $v(S)$ equals the weighted matroid rank function $r_{g}(S)$, i.e., the maximum $g$-weight of an independent subset of $S$.

Assignment valuations and weighted matroid rank functions are well-known examples of $\mathrm{M}^{\natural}$ concave (and, according to Theorem 2.3, gross substitutes) functions. This is true in general for Rado valuations.

Lemma 2.5. Every Rado valuation $v: 2^{\mathcal{G}} \rightarrow \mathbb{R}_{+}$is an $M^{\natural}$-concave function.

The proof is given in Section 7, using a more general construction by Murota [50]. It is worth noting that in 2003, Frank posed the question on whether the converse is also true: is the class of $\mathrm{M}^{\natural}$-concave functions the same as those of Rado valuations? ${ }^{3}$ In Section 7.3 we use an example from [45] showing that this is not the case. The main underlying reason is that this class is not minor closed. We then formulate a refined conjecture, and mention an earlier conjecture by Ostrovsky and Paes Leme [59], partially refuted by Tran [67].

\subsection{Continuous valuation functions}

The valuation functions $v$ in the Nash social welfare problem are defined on subsets of $\mathcal{G}$. Our arguments are based on convex relaxations, which requires a continuous extension of the valuation functions to $\mathbb{R}_{+}^{\mathcal{G}}$. We provide such an extension for Rado valuations; however, we note that a suitable extension does not even exist for general submodular valuations.

By a continuous valuation function we mean a continuous function $v:[0,1]^{\mathcal{G}} \rightarrow \mathbb{R}_{+}$with $v(\mathbf{0})=0$. We slightly abuse the notation by using $v$ to denote both discrete and continuous valuations; the value of a subset $S \subseteq \mathcal{G}$ of items will be $v\left(\chi_{S}\right)=v(S)$. Extending notions from discrete valuations, a function $f: \mathbb{R}_{+}^{\mathcal{G}} \rightarrow \mathbb{R}_{+}$is monotone if $f(x) \leq f(y)$ for $x \leq y, x, y \in \mathbb{R}_{+}^{\mathcal{G}}$, and subadditive if $f(x+y) \leq f(x)+f(y)$ for any $x, y \in[0,1]^{\mathcal{G}}$ such that $x+y \in[0,1]^{\mathcal{G}}{ }^{4}$

Whereas our overall result requires the continuous extension of Rado valuations, much weaker assumptions suffice for most parts of the argument, as formulated next.

Assumption 1. For every agent $i \in \mathcal{A}$ the continuous valuation function $v_{i}:[0,1]^{\mathcal{G}} \rightarrow \mathbb{R}_{+}$is monotone, concave, and subadditive.

\footnotetext{
${ }^{3}$ Personal communication by András Frank. See also Kazuo Murota's lecture [51], the problem sheet [52], and Renato Paes Leme's lecture [46].

${ }^{4}$ The more precise definition would be $f(x \vee y) \leq f(x)+f(y)$ for any $x, y \in[0,1]^{\mathcal{G}}$, where $x \vee y$ is the pointwise maximum of the vectors $x$ and $y$. For monotone valuations, these two definitions are equivalent.
} 
Concave extensions of discrete valuations For any discrete valuation function $v: 2^{\mathcal{G}} \rightarrow \mathbb{R}_{+}$, we can define the concave closure $\bar{v}:[0,1]^{\mathcal{G}} \rightarrow \mathbb{R}_{+}$as

$$
\bar{v}(x):=\min _{p \in \mathbb{R}^{\mathcal{G}}, \alpha \in \mathbb{R}}\{\langle p, x\rangle+\alpha \quad \text { s.t. } \quad p(S)+\alpha \geq v(S), \quad \forall S \subseteq \mathcal{G}\}
$$

see e.g. [50, Section 3.4]. As the minimum of linear functions, $\bar{v}$ is always concave. Note that it provides the concave upper envelope of the function $v$ defined on the discrete set $\{0,1\}^{\mathcal{G}}$, meaning that $\bar{v} \leq f$ for every concave function $f: \mathbb{R}_{+}^{\mathcal{G}} \rightarrow \mathbb{R}_{+}$such that $v(S) \leq f\left(\chi_{S}\right)$ for all $S \subseteq \mathcal{G}$.

We leave it to the reader as an exercise to verify that for an additive valuation $v(S)=\sum_{j \in S} v_{j}$, the concave closure is the linear function $\bar{v}(x)=\langle v, x\rangle$.

Whereas the extension $\bar{v}$ can be defined and is concave for every valuation function $v$, evaluating $\bar{v}(x)$ can be a hard problem. For example, in the case of submodular valuations, deciding whether $p(S)+\alpha \geq v(S)$ holds for all $S \subseteq \mathcal{G}$ amounts to submodular maximization and is thus NP-hard. Computing $\bar{v}(x)$ amounts to minimization over a polyhedron $P$ where separation is NP-hard; by the polynomial equivalence of optimization and separation [32], it follows that evaluating $\bar{v}(x)$ is NP-hard for submodular functions (see also [36, Lemma 6.15]).

Apart from computational hardness, another problem is that $\bar{v}\left(\chi_{S}\right)>v(S)$ may be possible for $S \subseteq \mathcal{G}$. If $\bar{v}\left(\chi_{S}\right)=v(S)$ for all subsets $S \subseteq \mathcal{G}$, then we say that $\bar{v}$ is the concave extension of $v$, and that $v$ is concave extensible.

Theorem 6.43 in [50] asserts that all $\mathrm{M}^{\natural}$-concave functions are concave extensible, and the converse is also essentially true. This underlines the importance of gross substitutes/ $\mathrm{M}^{\natural}$-concave valuations for our approach: this is the subclass of valuations where we can naturally use convex relaxation techniques. We also note that for $\mathrm{M}^{\mathrm{b}}$-concave functions, the concave extension can be evaluated in polynomial time. This is since, in contrast with general submodular functions, $\mathrm{M}^{\natural}$-concave functions can be efficiently maximized with a simple greedy algorithm.

The concave extension of Rado valuations For the case of Rado valuations, we now give an explicit description of the concave extension by a linear program. This representation of the concave extension is at the core of the arguments in Section 5, where we argue about the existence of a sparse optimal solution of a particular convex program.

Theorem 2.6. Consider a Rado valuation $v: 2^{\mathcal{G}} \rightarrow \mathbb{R}_{+}$given by a bipartite graph $(\mathcal{G}, V ; E)$ with costs on the edges $c: E \rightarrow \mathbb{R}_{+}$, and a matroid $\mathcal{M}=(V, \mathcal{I})$ with a rank function $r=r_{\mathcal{M}}$ as in Definition 2.4. For $x \in[0,1]^{\mathcal{G}}$, let us define

$$
\begin{array}{cc}
\nu(x):=\max \sum_{(j, k) \in E} c_{j k} z_{j k} & \\
\text { s.t.: } \quad \sum_{k \in V} z_{j k} \leq x_{j} \quad \forall j \in \mathcal{G} \\
\sum_{j \in \mathcal{G}, k \in T} z_{j k} \leq r(T) \quad \forall T \subseteq V \\
z \geq 0 .
\end{array}
$$

Then, $\nu=\bar{v}$ is the concave extension of $v$, and satisfies Assumption 1.

Proof. The function $\nu$ is clearly continuous and $\nu(\mathbf{0})=0$, thus, it is a valuation function. We postpone the proof that $\nu$ is the concave closure, i.e. $\nu=\bar{v}$ to Lemma 7.2. Let us now show that $\nu$ is a concave extension, namely $\nu\left(\chi_{S}\right)=v(S)$ for every $S \subseteq \mathcal{G}$. First, note that whenever $M^{\prime}$ is a feasible matching in the definition of $v(S), \chi_{M^{\prime}}$ is a feasible solution to (2) defining $\nu\left(\chi_{S}\right)$. The 
left hand side of the program defining $\nu\left(\chi_{S}\right)$ is integral, and the feasible region of (2) is a linear maximization problem over the intersection of two integral submodular polytopes on $E$. Using the total-dual integrality of polymatroid intersection, see [65, Theorem 46.1], the existence of an integer optimal solution $z \in \mathbb{Z}^{E}$ is guaranteed. Noting that $r(\{v\}) \leq 1$ for every $v \in V$, it follows that $z=\chi_{M}$ for a matching $M$, and $\delta_{V}(M)$ is independent in $\mathcal{M}$. We conclude that $\nu\left(\chi_{S}\right)=v(S)$.

Let us now turn to Assumption 1. Monotonicity is immediate. Concavity is implied by Lemma 7.2 , but let us also give a simple direct proof. Let $x, y \in \mathbb{R}_{+}, \lambda \in[0,1]$, and let $z$ and $z^{\prime}$ be the optimal solutions in the definition of $\nu(x)$ and $\nu(y)$. Then, it is immediate that $\lambda z+(1-\lambda) z^{\prime}$ is a feasible solution for the program defining $\nu(\lambda x+(1-\lambda) y)$, showing that $\nu(x)+\nu(y) \leq \nu(\lambda x+(1-\lambda) y)$.

For subadditivity, if $z$ is the optimal solution in the program defining $\nu(x+y)$ for some $x, y \in$ $[0,1]^{\mathcal{G}}$ with $x+y \in[0,1]^{\mathcal{G}}$, then we can easily decompose $z=z^{\prime}+z^{\prime \prime}$ such that $z^{\prime}$ is feasible to the program defining $\nu(x)$ and $z^{\prime \prime}$ if feasible for $y$. Thus, $\nu(x+y) \leq \nu(x)+\nu(y)$ follows.

In the light of this theorem, in the rest of the paper we will denote by $v:[0,1]^{\mathcal{G}} \rightarrow \mathbb{R}_{+}$the continuous Rado valuation defined in (2).

\subsection{Simple upper bounds}

We will often use the following simple bounds.

Lemma 2.7. Let $n, c \in \mathbb{N}, S \subseteq[n]$, and $1 \leq w_{1}, \ldots, w_{n} \leq \gamma-1$. For $i \in S$ let $k_{i} \in \mathbb{R}_{+}$such that $\sum_{i \in S} k_{i} \leq c \cdot n$. Then

$$
\left(\prod_{i \in S} k_{i}^{w_{i}}\right)^{1 / \sum_{i=1}^{n} w_{i}} \leq c \cdot \gamma .
$$

Proof. By the inequality of weighted arithmetic and geometric means we have:

$$
\begin{aligned}
\left(\prod_{i \in S} k_{i}^{w_{i}}\right)^{1 / \sum_{i=1}^{n} w_{i}} & =\prod_{i \in S} k_{i}^{\sum_{i=1}^{n} w_{i}} \cdot \prod_{i \in[n] \backslash S} 1^{\frac{w_{i}}{\sum_{i=1}^{n} w_{i}}} \\
& \leq \sum_{i \in S} \frac{w_{i} k_{i}}{\sum_{i=1}^{n} w_{i}}+\sum_{i \in[n] \backslash S} \frac{w_{i}}{\sum_{i=1}^{n} w_{i}} \leq(\gamma-1) \frac{\sum_{i \in S} k_{i}}{\sum_{i=1}^{n} w_{i}}+1 \leq c \cdot \gamma .
\end{aligned}
$$

Lemma 2.8. Let $n, c \in \mathbb{N}, S \subseteq[n]$. For $i \in S$ let $k_{i} \in \mathbb{R}_{+}$such that $\sum_{i \in S} k_{i} \leq c \cdot n$. Then

$$
\left(\prod_{i \in S} k_{i}\right)^{1 / n} \leq c \cdot e^{1 / e}
$$

Proof. We present the proof for $c=1$, the general case easily reduces to $c=1$ by scaling. Without loss of generality, we assume that $k_{i} \geq 1$ for $i \in S$. The worst case happens when $\sum_{i \in S} k_{i}=n$. For fixed size of $S$, the product $\prod_{i \in S} k_{i}$ is maximized when all $k_{i}$ are the same. Hence, $\left(\prod_{i \in S} k_{i}\right)^{1 / n} \leq$ $\left(\frac{n}{|S|}\right)^{|S| / n}$. Let $\xi=\frac{n}{|S|}$ then $\left(\frac{n}{|S|}\right)^{|S| / n}=\xi^{1 / \xi}$. By the first order conditions, the value $\xi^{1 / \xi}$ achieves the maximum for $\xi=e$. Hence, $\left(\prod_{i \in S} k_{i}\right)^{1 / n} \leq e^{1 / e}$. 


\section{Overview of the approach}

Let $v_{i}$ be a continuous valuation function and $w_{i}>0$ be the weight for each $i \in \mathcal{A}$. Given a fractional allocation $x=\left(x_{1}, \ldots, x_{n}\right) \in \mathbb{R}_{+}^{\mathcal{A} \times \mathcal{G}}$, we let

$$
\operatorname{NSW}(x):=\left(\prod_{i \in \mathcal{A}} v_{i}\left(x_{i}\right)^{w_{i}}\right)^{1 / \sum_{i} w_{i}}
$$

Then, the asymmetric Nash social welfare program is captured by the following integer program.

$$
\max \operatorname{NSW}(x) \quad \text { s.t. } \sum_{i \in \mathcal{A}} x_{i j} \leq 1 \forall j \in \mathcal{G}, x \in\{0,1\}^{E} .
$$

Let OPT denote the optimum value. The natural relaxation is (NSW-IP) is

$$
\max \operatorname{NSW}(x) \quad \text { s.t. } \sum_{i \in \mathcal{A}} x_{i j} \leq 1 \forall j \in \mathcal{G}, x \geq 0 .
$$

The objective is log-concave assuming the $v_{i}$ 's are concave functions. However, Cole and Gkatzelis [17, Lemma 3.1] showed that this relaxation has unbounded integrality gap already for additive valuations.

We propose a mixed integer programming relaxation instead of (3). Consider a set of items $\mathcal{H} \subseteq \mathcal{G}$. Our mixed relaxation requires the items in $\mathcal{H}$ to be allocated integrally and the rest can be allocated fractionally.

$$
\begin{array}{rlrl}
\max & \operatorname{NSW}(x) & \\
\text { s.t.: } \quad \sum_{i \in \mathcal{A}} x_{i j} & \leq 1 \quad \forall j \in \mathcal{G} \\
x_{i j} & \in\{0,1\} \quad \forall j \in \mathcal{H}, \forall i \in \mathcal{A} \\
x & \geq 0 .
\end{array}
$$

(Mixed relaxation)

This clearly gives a relaxation of $(\mathrm{NSW}-\mathrm{IP}): \mathrm{OPT}_{\mathcal{H}} \geq \mathrm{OPT}$ where $\mathrm{OPT}_{\mathcal{H}}$ is optimal value of (Mixed relaxation) for any set of items $\mathcal{H}$. Theorem 1.2 is shown by constructing an integer allocation $x \in\{0,1\}^{\mathcal{A} \times \mathcal{G}}$ and an item set $\mathcal{H}$ such that $\mathrm{NSW}(x) \geq \mathrm{OPT}_{\mathcal{H}} /\left(256 \gamma^{3}\right)$. This is proved in five phases:

Phase I Find an appropriate item set $\mathcal{H}$.

Phase II Approximate (Mixed relaxation) by another integer program (Mixed+matching).

Phase III Find an approximate mixed integer solution to (Mixed+matching).

Phase IV Find a sparse approximate mixed integer solution to (Mixed+matching).

Phase V Round the mixed integer solution to an integer solution.

We note that phases are not necessarily algorithmic phases but also conceptional reductions of the problem. Regardless, we call them phases for the sake of presentation. We now give an overview of all the phases; most proofs are deferred to later sections. 


\subsection{Phase I: Finding the item set $\mathcal{H}$}

We solve a maximum weight matching problem that achieves the highest Nash social welfare value under the restriction that each agent may only receive a single item. This can be achieved by assigning an edge weight $\omega_{i j}=w_{i} \log \left(v_{i j}\right)$ for every $i \in \mathcal{A}, j \in \mathcal{G}$, and solving the maximum weight assignment problem in the complete bipartite graph between $\mathcal{A}$ and $\mathcal{G}$; we recall the notation $v_{i j}=v_{i}(\{j\})$. We let $\tau: \mathcal{A} \rightarrow \mathcal{G}$ denote the optimal matching represented as a mapping, i.e. $\tau(i)$ is the item matched to agent $i \in \mathcal{A}$. We define $\mathcal{H}$ as the set of items assigned by $\tau$, i.e., $\mathcal{H}:=\tau(\mathcal{A})$. We will refer to this set $\mathcal{H}$ as the set of most preferred items. ${ }^{5}$

The existence of $\tau$ with finite weight proves that the instance is feasible, i.e., there is a way of allocating one item to each agent such that agent values the assigned item positively. On the other hand, if no finite weight matching exists, the optimum value to (NSW-IP) is 0. Henceforth, we assume without loss of generality that the optimal NSW is non-zero.

\subsection{Phase II: Reduction to the mixed matching relaxation}

We approximate (Mixed relaxation) by a second mixed integer program. We use variables $y \in$ $\mathbb{R}_{+}^{\mathcal{A} \times(\mathcal{G} \backslash \mathcal{H})}$ representing the fractional allocations of the items in $\mathcal{G} \backslash \mathcal{H}$. Even though the valuation functions $v_{i}$ are defined on $\mathbb{R}_{+}^{\mathcal{G}}$, we use $v_{i}\left(y_{i}\right)$ to denote $v_{i}\left(x_{i}\right)$, where $x_{i}$ is obtained from $y_{i}$ by setting $x_{i j}=0$ for $j \in \mathcal{H}$ and $x_{i j}=y_{i j}$ for $j \in \mathcal{G} \backslash \mathcal{H}$.

$$
\begin{array}{cc}
\max & \left(\prod_{i \in \mathcal{A}}\left(v_{i}\left(y_{i}\right)+v_{i \sigma(i)}\right)^{w_{i}}\right)^{1 / \sum_{i} w_{i}} \\
\text { s.t.: } & \sum_{i \in \mathcal{A}} y_{i j} \leq 1 \quad \forall j \in \mathcal{G} \backslash \mathcal{H} \quad \text { (Mixed+matching) } \\
& y_{i j} \geq 0 \\
\sigma: \mathcal{A} \rightarrow \mathcal{H} \text { is a matching. } & \forall j \in \mathcal{G} \backslash \mathcal{H}, \forall i \in \mathcal{A}
\end{array}
$$

We will refer to this program as the mixed matching relaxation. The program (Mixed+matching) differs from (Mixed relaxation) in two respects. Firstly, the objective differs from NSW $(x)$ : for each agent, the value of each agent in (Mixed relaxation) is given by the Rado valuation while in (Mixed+matching) we evaluate the utility of each agent separately on $\mathcal{H}$ and $\mathcal{G} \backslash \mathcal{H}$ and take the sum of these two values. Secondly, and more importantly, we require that the items in $\mathcal{H}$ are allocated to the agents by a matching. Unlike (Mixed relaxation), this will not be a relaxation of (NSW-IP): the optimal integer solution may allocate multiple items in $\mathcal{H}$ to the same agent. We show that the effect of both these changes is limited.

Let $(y, \sigma)$ be a feasible solution to (Mixed+matching). We define $\overline{\mathrm{NSW}}(y, \sigma)$ as the objective function value in (Mixed+matching), and let $\overline{\mathrm{OPT}}_{\mathcal{H}}$ denote the optimum value. Let us define $\operatorname{NSW}(y, \sigma)$ as the Nash social welfare of the same allocation. Namely, NSW $(y, \sigma)=\operatorname{NSW}(x)$, where $x_{i j}=y_{i j}$ if $j \in \mathcal{G} \backslash \mathcal{H}$, and for $j \in \mathcal{H}$ we have $x_{i j}=1$ if $j=\sigma(i)$, and $x_{i j}=0$ otherwise. The next lemma is an easy consequence of monotonicity and subadditivity.

Lemma 3.1. For any feasible solution $(y, \sigma)$ to (Mixed+matching), we have

$$
\overline{\mathrm{NSW}}(y, \sigma) \geq \mathrm{NSW}(y, \sigma) \geq \frac{1}{2} \overline{\mathrm{NSW}}(y, \sigma) .
$$

Proof. We have $\overline{\mathrm{NSW}}(y, \sigma) \geq \mathrm{NSW}(y, \sigma)$ by subadditivity. By monotonicity: $2 \mathrm{NSW}(y, \sigma) \geq$ $\operatorname{NSW}(y, \emptyset)+\operatorname{NSW}(0, \sigma)=\overline{\operatorname{NSW}}(y, \sigma)$.

\footnotetext{
${ }^{5}$ Interestingly, in case of symmetric agents endowed with additive valuations the set $\mathcal{H}$ contains all items with price at least one in any spending restricted equilibrium as in [17]; see Section 8.
} 
Using this lemma, as well as Lemma 2.7, we can relate the optimum values and approximate solutions of (Mixed relaxation) and (Mixed+matching).

Theorem 3.2. Let $\mathcal{H} \subseteq \mathcal{G}$ with $|\mathcal{H}|=|\mathcal{A}|$. For the optimum values $\mathrm{OPT}_{\mathcal{H}}$ to (Mixed relaxation) and $\overline{\mathrm{OPT}}_{\mathcal{H}}$ to (Mixed+matching), we have

$$
\overline{\mathrm{OPT}}_{\mathcal{H}} \geq \frac{1}{\gamma} \mathrm{OPT}_{\mathcal{H}}
$$

Let $(y, \sigma)$ be an $\alpha$-approximate optimal solution to (Mixed+matching), that is, $\overline{\mathrm{NSW}}(y, \sigma) \geq$ $\frac{1}{\alpha} \overline{\mathrm{OPT}}_{\mathcal{H}}$. Then, $\operatorname{NSW}(y, \sigma) \geq \frac{1}{2 \alpha \gamma} \mathrm{OPT}_{\mathcal{H}}$. If the valuation functions $v_{i}$ are additive, then the stronger bound $\mathrm{NSW}(y, \sigma) \geq \frac{1}{\alpha \gamma} \mathrm{OPT}_{\mathcal{H}}$ applies.

Proof. We first show that $\overline{\mathrm{OPT}}_{\mathcal{H}} \geq \frac{1}{\gamma} \mathrm{OPT}_{\mathcal{H}}$. Let $x$ be an optimal solution to (Mixed relaxation). For each agent $i$, let $K_{i}$ be the set of items agent $i$ receives from $\mathcal{H}$ under $x$; and let $y$ be the restriction of $x$ on $\mathcal{G} \backslash \mathcal{H}$ defined as $y_{i j}=x_{i j}$ for $j \in \mathcal{G} \backslash \mathcal{H}$ and $y_{i j}=0$ otherwise. Let $k_{i}:=\left|K_{i}\right|$. Denote with $S$ the set of agents that receive at least one items from $\mathcal{H}$, i.e., $S=\left\{i \in \mathcal{A}: k_{i} \geq 1\right\}$. For each agent $i \in S$ let $\sigma(i)=\max _{j \in K_{i}}\left\{v_{i j}\right\}$, and define $\sigma(i)=\emptyset$ for $i \in \mathcal{A} \backslash S$. Then, $(y, \sigma)$ is a feasible solution of (Mixed+matching). In other words, $(y, \sigma)$ is obtained from $x$ once each agent $i \in S$ discards all items from $K_{i}$ except the most valuable one. By monotonicity and subadditivity, for all $i \in S$, we have

$$
v_{i}\left(x_{i}\right) \leq v_{i}(y)+\sum_{j \in K_{i}} v_{i j} \leq k_{i} \cdot\left(v_{i}(y)+v_{i \sigma(i)}\right) .
$$

Therefore,

$$
\frac{\operatorname{OPT}_{\mathcal{H}}}{\overline{\operatorname{OPT}}_{\mathcal{H}}} \leq \frac{\mathrm{NSW}(x)}{\overline{\operatorname{NSW}}(y, \sigma)}=\left(\prod_{i \in S} \frac{v_{i}\left(x_{i}\right)^{w_{i}}}{\left(v_{i}(y)+v_{i \sigma(i)}\right)^{w_{i}}}\right)^{1 / \sum_{i} w_{i}} \leq\left(\prod_{i \in S} k_{i}^{w_{i}}\right)^{1 / \sum_{i} w_{i}}
$$

Moreover, $\sum_{i \in S} k_{i} \leq|\mathcal{H}|=|\mathcal{A}|=n$. Then, the bound follows by Lemma 2.7. The second part of the theorem follows by Lemma 3.1.

\subsection{Phase III: Approximating the mixed matching relaxation}

Our next goal is to find a 2-approximation solution to (Mixed+matching); we do not know whether this problem is polynomial-time solvable. By Theorem 3.2, this yields a $(4 \gamma)$-approximation to (Mixed relaxation).

Let us first remove all items in $\mathcal{H}$. Some agents may only value positively the items $\mathcal{H}$. We let $\mathcal{A}^{\prime}$ the subset of agents who have positive values for the items $\mathcal{G} \backslash \mathcal{H}$, that is, $\mathcal{A}^{\prime}:=\{i \in \mathcal{A}$ : $\left.v_{i}(\mathcal{G} \backslash \mathcal{H})>0\right\}$. Consider the "naïve" relaxation (3) on the instance restricted to $\mathcal{A}^{\prime}$ and $\mathcal{G} \backslash \mathcal{H}$, and taking the logarithm of the objective

$$
\begin{array}{cc}
\max & \sum_{i \in \mathcal{A}^{\prime}} w_{i} \log \left(v_{i}\left(y_{i}\right)\right) \\
\text { s.t.: } & \sum_{i \in \mathcal{A}^{\prime}} y_{i j} \leq 1 \quad \forall j \in \mathcal{G} \backslash \mathcal{H} \\
y \geq 0 .
\end{array}
$$

This is the classical Eisenberg-Gale convex program that computes an equilibrium in Fisher markets with divisible items for homogeneous concave valuation functions [22]. Given an optimal solution $y^{*} \in \mathbb{R}_{+}^{\mathcal{A}^{\prime} \times(\mathcal{G} \backslash \mathcal{H})}$ of $(\mathrm{EG})$ we can find an approximate solution to (Mixed+matching). 
Theorem 3.3. Let $\mathcal{H} \subseteq \mathcal{G}$ with $|\mathcal{H}|=|\mathcal{A}|$. Let $\pi^{*}$ be maximum weight assignment in the complete bipartite graph between $\mathcal{A}$ and $\mathcal{H}$, with edge weights $\omega_{i j}=w_{i} \log \left(v_{i}\left(y_{i}^{*}\right)+v_{i j}\right)$ for $i \in \mathcal{A}, j \in \mathcal{H}$. Then, $\overline{\mathrm{NSW}}\left(y^{*}, \pi^{*}\right) \geq \frac{1}{2} \overline{\mathrm{OPT}}_{\mathcal{H}}$.

Theorem 3.3 is an immediate consequence of the following lemma.

Lemma 3.4. Let $\mathcal{H} \subseteq \mathcal{G}$ with $|\mathcal{H}|=|\mathcal{A}|$. Let $\alpha>0$ and $y^{*}$ be an optimal and $y$ a feasible solution of (EG) such that $v_{i}\left(y_{i}\right) \geq \frac{1}{\alpha} v_{i}\left(y_{i}^{*}\right)$ for all $i \in \mathcal{A}^{\prime}$. Let $\pi$ be maximum weight assignment in the bipartite graph with colour classes $\mathcal{A}$ and $\mathcal{H}$, and edge weights $\omega_{i j}=w_{i} \log \left(v_{i}\left(y_{i}\right)+v_{i j}\right)$ for $i \in \mathcal{A}$, $j \in \mathcal{H}$. Then,

$$
\overline{\operatorname{NSW}}(y, \pi) \geq \frac{1}{2 \alpha} \overline{\mathrm{OPT}}_{\mathcal{H}} .
$$

Since valuations $v_{i}$ are concave, (EG) is a convex program. For any $\varepsilon>0$, we can find an $(1-\varepsilon)$-approximate solution in polynomial-time, where the running time depends on $\log (1 / \varepsilon)$. It turns out that approximation of the objective function might not be enough. In Lemma 3.4 we require an agent-wise approximate solution: each agent gets at least a constant fraction of her value in the optimum. It is not clear if finding such agent-wise approximation is possible in polynomial time for general concave valuations $v_{i}$, but as we will see in the next section we can find an exact optimal solution for Rado valuations.

The proof of Lemma 3.4 is deferred to Section 4. It does not depend on the choice of $\mathcal{H}$ but only requires $|\mathcal{H}|=|\mathcal{A}|$.

\subsection{Phase IV: A sparse approximate solution for the mixed matching relaxation}

In this section we exploit the properties of Rado valuations. Assuming the agents have Rado valuation functions, we can find an approximate solution of (Mixed+matching) with a strong sparsity property. Even though the approximation ratio is weaker than given in Theorem 3.3, sparsity will be essential for the rounding in Phase V.

Theorem 3.5. Suppose the functions vi are Rado valuations. Let $\mathcal{H} \subseteq \mathcal{G}$ with $|\mathcal{H}|=|\mathcal{A}|$. We can find a feasible solution $(y, \pi)$ to (Mixed+matching) such that

(i) $\overline{\mathrm{NSW}}(y, \pi) \geq \frac{1}{4} \overline{\mathrm{OPT}}_{\mathcal{H}}$,

(ii) $\operatorname{supp}(y) \leq 2|\mathcal{A}|+\left|\mathcal{L}^{+}\right|$where $\mathcal{L}^{+}=\left\{j \in \mathcal{G} \backslash \mathcal{H}: \sum_{i \in \mathcal{A}^{\prime}} y_{i j}>0\right\}$, that is, $\mathcal{L}^{+}$is the set of allocated items in $y$.

Moreover, for additive valuation functions, we can strengthen (i) to $\mathrm{NSW}(y, \sigma) \geq \frac{1}{2} \mathrm{OPT}_{\mathcal{H}}$ and (ii) to $\operatorname{supp}(y) \leq|\mathcal{A}|+\left|\mathcal{L}^{+}\right|$.

Let us start with the special case of additive valuations. In this case, an exact solution $y^{*}$ to the Eisenberg-Gale convex program (EG) can be found in strongly polynomial time [58, 70].

Theorem 3.6. Assuming the valuations $v_{i}$ are additive, we can find an optimal solution $y^{*}$ of (EG) in strongly polynomial time such that the support $\operatorname{supp}\left(y^{*}\right)$ is a forest.

The claim on the support follows easily by showing that any cycles in $\operatorname{supp}\left(y^{*}\right)$ can be eliminated, see e.g., $[17,21,58]$. Consequently, $\left|\operatorname{supp}\left(y^{*}\right)\right| \leq\left|\mathcal{A}^{\prime}\right|+\left|\mathcal{L}^{+}\right|-1$. Together with Lemma 3.4, this proves the statement in Theorem 3.5 for additive valuations.

For Rado valuations, we first prove that an optimal solution of (EG) can be found in polynomial time, see Section 5.1. We first show that this is a rational convex program, and use the variant of the ellipsoid method for rational polyhedron [32]. 
Lemma 3.7. Suppose that for each agent $i \in \mathcal{A}, v_{i}$ is a Rado valuation given by a bipartite graph $\left(\mathcal{G}, V_{i} ; E_{i}\right)$, integer costs $c_{i}: E_{i} \rightarrow \mathbb{Z}$ and a matroid $\mathcal{M}_{i}=\left(V_{i}, \mathcal{I}_{i}\right)$ as in Definition 2.4. Let $T=\max _{i \in \mathcal{A}}\left|V_{i}\right|$, and $C=\max _{i \in \mathcal{A}}\left\|c_{i}\right\|_{\infty}$. Let the weights $w_{i}>0$ be rational numbers given as quotients of two integers at most $U$. Assume the matroids $\mathcal{M}_{i}$ are given by rank oracles. Then, (EG) has a rational solution with $\operatorname{poly}(|\mathcal{A}|,|\mathcal{G}|, T, \log C, \log U)$ bit-complexity, and such a solution can be found in $\operatorname{poly}(|\mathcal{A}|,|\mathcal{G}|, T, \log C, \log U)$ arithmetic operations and calls to the matroid rank oracles.

Our next lemma shows that any feasible solution to (EG) can be sparsified by losing at most the half of the value for each agent, see Section 5.2. This is achieved in two steps, using the sparsity of basic feasible solutions to linear programs. Half of the valuation may be lost in the second step, where for the fractionally allocated items we aim to remove one of the fractional edges. The set to be deleted is identified by writing an auxiliary linear program.

Lemma 3.8. Suppose the functions $v_{i}$ are Rado valuations, and let $\hat{y}$ be a feasible solution to (EG). Then, in polynomial time we can find a feasible solution y such that

(i) $v_{i}(y) \geq \frac{1}{2} v_{i}(\hat{y})$,

(ii) $|\operatorname{supp}(y)| \leq 2\left|\mathcal{A}^{\prime}\right|+\left|\mathcal{L}^{+}\right|$where $\mathcal{L}^{+}:=\mathcal{L}^{+}(y)=\left\{j \in \mathcal{G} \backslash \mathcal{H}: \sum_{i \in \mathcal{A}^{\prime}} y_{i j}>0\right\}$.

By combining Lemmas 3.4, 3.7, 3.8, we obtain Theorem 3.5 for Rado valuations.

\subsection{Phase V: Rounding the mixed integer solution}

For this phase of the algorithm, we require a sparse approximate solution as in Theorem 3.5, and exploit the choice of $\mathcal{H}$ as the set of most preferred items in Phase I. We start with a mixed integer solution $(y, \pi)$ as in Theorem 3.5. By a reduction of $(y, \pi)$ we mean a mixed integer solution $\left(y^{r}, \pi\right)$ obtained as follows. For each $j \in \mathcal{L}^{+}$, we pick an arbitrary agent $\kappa(j) \in \mathcal{A}$ such that $y_{\kappa(j) j}>0$. We set $y_{\kappa(j) j}^{r}=y_{\kappa(j) j}$, and set $y_{i j}^{r}=0$ if $i \neq \kappa(j)$. By the bound on $\operatorname{supp}(y)$, this amounts to setting $\leq 2|\mathcal{A}|$ values $y_{i j}$ to 0 . The proof of the next lemma is given in Section 6 .

Lemma 3.9. Let $\mathcal{H}$ be the set of most preferred items, and let $(y, \pi)$ be a solution to (Mixed+matching) as in Theorem 3.5. Let $\left(y^{r}, \pi\right)$ be a reduction of $(y, \pi)$. Then in polynomial-time we can find a matching $\rho: \mathcal{A} \rightarrow \mathcal{H}$ such that

$$
\overline{\mathrm{NSW}}\left(y^{r}, \rho\right) \geq \frac{1}{32 \gamma^{2}} \overline{\mathrm{NSW}}(y, \pi) .
$$

Further, if the valuations are linear, then we can find a matching $\rho: \mathcal{A} \rightarrow \mathcal{H}$ such that $\overline{\mathrm{NSW}}\left(y^{r}, \rho\right) \geq$ $\frac{1}{8} \overline{\mathrm{NSW}}(y, \pi)$.

Such a matching $\rho$ can be found by combining the matching $\pi$ in the solution $(y, \pi)$, and the initial matching $\tau$ from Phase I that delivers the highest NSW value such that every agent may receive only one item. We swap from $\pi$ to $\tau$ on certain alternating paths and cycles.

We are ready to prove the main results.

Theorem 1.2 (Main). There exists a polynomial-time $256 \gamma^{3}$-approximation algorithm for the Nash social welfare problem with Rado valuation functions. For additive valuation functions, there exists a polynomial-time $16 \gamma$-approximation algorithm. 
Proof. From Theorem 3.5 and Lemma 3.9, we can obtain a solution an $\left(128 \gamma^{2}\right)$-approximate solution $\left(y^{r}, \rho\right)$ to (Mixed+matching) such that for each item $\mathcal{L}^{+}$there is exactly one incident edge in $\operatorname{supp}\left(y^{r}\right)$. We can obtain a $0-1$ valued solution $x$ to (NSW-IP) by assigning each item in $\mathcal{H}$ according to $\rho$ and each item $j \in \mathcal{L}^{+}$to the unique agent $i$ with $y_{i j}^{r}>0$. Clearly, NSW $(x) \geq \operatorname{NSW}\left(y^{r}, \rho\right)$. We obtain $\operatorname{NSW}(x) \geq \mathrm{OPT}_{\mathcal{H}} /\left(256 \gamma^{3}\right) \geq \mathrm{OPT} /\left(256 \gamma^{3}\right)$ using Theorem 3.2. For additive valuations, we use the stronger bounds in the same results.

Theorem 1.1. There exists a polynomial-time $256 e^{3 / e} \approx 772$-approximation algorithm for the symmetric Nash social welfare problem under Rado valuations.

Proof. The proof follows exactly as the proof of Theorem 1.2 once we replace $\gamma$ by $e^{1 / e}$. Such a change is justified as in the symmetric case we can use Lemma 2.8 instead of the bound given by Lemma 2.7.

\section{Phase III: Approximating the mixed matching relaxation}

Phase III presents a general way of obtaining a 2-approximation to (Mixed+matching). By Theorem 3.2, this gives a $(4 \gamma)$-approximation to (Mixed relaxation), a mixed integer relaxation of the ANSW problem. Recall that (Mixed+matching) is the following mixed integer program

$$
\begin{array}{cc}
\max & \left(\prod_{i \in \mathcal{A}}\left(v_{i}\left(y_{i}\right)+v_{i \sigma(i)}\right)^{w_{i}}\right)^{1 / \sum_{i} w_{i}} \\
\text { s.t.: } & \sum_{i \in \mathcal{A}} y_{i j} \leq 1 \quad \forall j \in \mathcal{G} \backslash \mathcal{H} \quad \text { (Mixed+matching) } \\
& y_{i j} \geq 0 \\
& \sigma: \mathcal{A} \rightarrow \mathcal{H} \text { is a matching. }
\end{array}
$$

In the above problem, we need to allocate items $\mathcal{G}$ to the agents in $\mathcal{A}$ in order to maximize an objective function that is an approximation of the NSW. Items in $\mathcal{G} \backslash \mathcal{H}$ can be allocated fractionally to the agents without any constraints. The items in $\mathcal{H}$ have to be allocated integrally via an assignment, thereby allocating exactly one item from $\mathcal{H}$ to each agent $\mathcal{A}$.

While the exact computational complexity of (Mixed+matching) remains unresolved, we show that we can 2-approximate it.

Denote $\mathcal{L}=\mathcal{G} \backslash \mathcal{H}$. Let $\mathcal{A}^{\prime}$ be the subset of agents that have positive value for the items in $\mathcal{G} \backslash \mathcal{H}, \mathcal{A}^{\prime}:=\left\{i \in \mathcal{A}: v_{i}(\mathcal{G} \backslash \mathcal{H})>0\right\}$, as some agents may only have positive value for the items in $\mathcal{H}$. Restricting (Mixed+matching) to the items $\mathcal{L}$ and agents $\mathcal{A}^{\prime}$ and taking the objective yields an instance of (EG):

$$
\begin{array}{ll}
\max & \sum_{i \in \mathcal{A}^{\prime}} w_{i} \log v_{i}\left(y_{i}\right) \\
\text { s.t.: } & \sum_{i \in \mathcal{A}^{\prime}} y_{i j} \leq 1 \quad \forall j \in \mathcal{L} \\
& y_{i j} \geq 0 \quad \forall j \in \mathcal{L}, \forall i \in \mathcal{A}^{\prime} .
\end{array}
$$

The above is a convex program whenever the valuations $v_{i}($.$) are concave, and we can solve it to an$ arbitrary precision in polynomial time if we have access to a supergradient oracle to the objective function.

On the other hand, suppose that the variables $y$ are fixed in (Mixed+matching). Under the fixed $y$, we can find an optimal assignment $\sigma$. Namely, an optimal assignment is exactly a maximum 
weight assignment in the bipartite graph $(\mathcal{A}, \mathcal{H} ; E)$ where the weight of an edge $i j$ for $i \in \mathcal{A}, j \in \mathcal{H}$ is $\omega_{i j}:=w_{i} \log \left(v_{i}\left(y_{i}\right)+v_{i j}\right)$.

Informally, (Mixed +matching) is a combination of two tractable problems. We show that an optimal solution $y^{*}$ to the restriction of the problem to $\mathcal{L}$ and $\mathcal{A}^{\prime}$, and an optimal assignment with respect to the fixed $y^{*}$ gives a 2 -approximation for (Mixed+matching).

In Section 4.1 we discuss the restriction of the problem to $\mathcal{L}$ and $\mathcal{A}^{\prime}$ and give a technical lemma. The main result of the section is presented in Section 4.2.

\subsection{Properties of Eisenberg-Gale program}

Let us now consider the Eisenberg-Gale program (EG). An optimal solution $y^{*}$ and the optimal Lagrange multipliers $p_{j}$ for $j \in \mathcal{L}$ can be interpreted as the so-called Gale equilibrium in the market with divisible items $\mathcal{L}$, agents $\mathcal{A}^{\prime}$, and where agent $i$ has valuation $v_{i}$ and budget $w_{i}$. In particular, $y^{*}$ represent the allocations and $p_{j}$ for $j \in \mathcal{L}$, specify the prices in the market equilibrium, see e.g., $[29,55] .^{6}$

Our technical lemma relates the combined difference in valuations of each agent in the optimal solution $y^{*}$ and any other allocation $y^{\prime}$. The rest of Section 4.1 is devoted to its proof.

Lemma 4.1. Let $y^{*}$ be an optimal solution to (EG). Then for any feasible solution $y^{\prime}$ and any $\mathcal{A}^{\prime \prime} \subseteq \mathcal{A}^{\prime}$ it holds

$$
\sum_{i \in \mathcal{A}^{\prime \prime}} w_{i} \frac{v_{i}\left(y_{i}^{\prime}\right)}{v_{i}\left(y_{i}^{*}\right)} \leq \sum_{i \in \mathcal{A}^{\prime \prime}} w_{i}+\sum_{i \in \mathcal{A}^{\prime}} w_{i} .
$$

We recall some definitions and the Karush-Kuhn-Tucker (KKT) optimality conditions in terms of subgradients; see [64, Chapter 2 and Theorem 3.27]. Given a convex function $f: \mathbb{R}^{M} \rightarrow \mathbb{R}$, we say that $g$ is a subgradient of $h$ at $y^{*} \in \mathbb{R}^{M}$ if $f(y) \geq f\left(y^{*}\right)+g^{\top}\left(y-y^{*}\right)$ for all $y \in \mathbb{R}^{M}$. The set of all subgradients at a point $y^{*}$ is called subdifferential and denoted as $\partial f\left(y^{*}\right)$. If the function is differentiable then $\partial f\left(y^{*}\right)=\left\{\nabla f\left(y^{*}\right)\right\}$. Consider the convex program

$$
\begin{array}{ll}
\min & f_{0}(y) \\
\text { s.t.: } & f_{j}(y) \leq 0 \quad \forall j \in \mathcal{L} \\
& y \geq 0,
\end{array}
$$

where $f_{j}$ for $j \in\{0\} \cup \mathcal{L}$ is convex. Assume that the there exists a strict feasible point (Slater's condition). Then, $y^{*}$ is a an optimal solution with the Lagrange multipliers $p$, if and only if the following conditions hold

- $f_{j}\left(y^{*}\right) \leq 0, p_{j} \geq 0$ for all $j \in \mathcal{L}$ (primal and dual feasibility),

- $0 \in \partial f_{0}\left(y^{*}\right)+\sum_{j \in \mathcal{L}} p_{j} \partial f_{j}\left(y^{*}\right)+\left\{\mu \in \mathbb{R}_{-}^{M}: \mu^{\top} y^{*}=0\right\}$ (stationarity), and

- $p_{j} f_{j}\left(y^{*}\right)=0$ (complementary slackness).

We say that $g$ is a supergradient of the concave function $f$ if $-g$ is a subgradient of $-f$. The following proposition guarantees the existence of supergradients.

Proposition 4.2. The function $f: \mathbb{R}_{+}^{M} \rightarrow \mathbb{R}$ is concave if and only if $\forall y^{*} \in \mathbb{R}_{+}^{M}$ it has a non-empty superdifferential at $y^{*}$. In other words, there is $g \in \mathbb{R}^{M}$ such that

$$
f(y) \leq f\left(y^{*}\right)+g^{\top}\left(y-y^{*}\right) .
$$

\footnotetext{
${ }^{6}$ In case of homogeneous valuations this can be used to find a Fisher equilibrium, since Fisher and Gale equilibria coincide under homogeneous valuations [23, 55].
} 
We can interpret the Lagrange multipliers in (EG) as prices; the next claim states that no agent spends more that her budget in a Gale-equilibrium.

Claim 4.3. Let $y^{*}$ be an optimum and $p$ be the optimal Lagrange multipliers of (EG). For all $i \in \mathcal{A}^{\prime}$ it holds $p^{\top} y_{i}^{*} \leq w_{i}$.

Proof. Let us apply the above KKT conditions to the concave maximization program (EG). The stationary condition can be written such that for each agent $i \in \mathcal{A}^{\prime}$

$$
0 \in \partial\left(-w_{i} \log \left(v_{i}\left(y_{i}^{*}\right)\right)\right)+p+\left\{\mu_{i} \in \mathbb{R}_{-}^{\mathcal{L}}: \mu_{i}^{\top} y_{i}^{*}=0\right\} .
$$

By the composition rules for subgradients we have

$$
0 \in-\frac{w_{i} \partial v_{i}\left(y_{i}^{*}\right)}{v_{i}\left(y_{i}^{*}\right)}+p+\left\{\mu_{i} \in \mathbb{R}_{-}^{\mathcal{L}}: \mu_{i}^{\top} y_{i}^{*}=0\right\}
$$

Therefore, there exists a supergradient $g_{i} \in \partial v_{i}\left(y_{i}^{*}\right)$ such that $w_{i} g_{i}^{\top}=v_{i}\left(y_{i}^{*}\right) \cdot\left(p^{\top}+\mu_{i}^{\top}\right)$ where $\mu_{i} \leq 0$ and $\mu_{i}^{\top} y_{i}^{*}=0$.

By definition of subgradient (supergradient) at $y_{i}^{*}$, we have that $g_{i}^{\top} y_{i}^{*} \leq v_{i}\left(y_{i}^{*}\right)$ for all $i \in \mathcal{A}^{\prime}$. It follows that $p^{\top} y_{i}^{*} \leq w_{i}$ for all $i \in \mathcal{A}^{\prime}$.

Proof of Lemma 4.1. By the KKT conditions, for each $i \in \mathcal{A}^{\prime}$, we have a supergradient $g_{i} \in \partial v_{i}\left(y_{i}^{*}\right)$ such that $\frac{w_{i} g_{i}}{v_{i}\left(y_{i}^{*}\right)} \leq p$ holds. By complementarity slackness, if $p_{j}>0$ then $\sum_{i \in \mathcal{A}^{\prime}} y_{i j}^{*}=1$. Let $\bar{y}_{i j}=\max \left\{y_{i j}^{*}, y_{i j}^{\prime}\right\}$. Then we obtain:

$$
v_{i}\left(y_{i}^{\prime}\right) \leq v_{i}\left(\bar{y}_{i}\right) \leq v_{i}\left(y_{i}^{*}\right)+g_{i}^{\top}\left(\bar{y}_{i}-y_{i}^{*}\right) \leq v_{i}\left(y_{i}^{*}\right)+\frac{v_{i}\left(y_{i}^{*}\right) p^{\top}}{w_{i}} \cdot\left(\bar{y}_{i}-y_{i}^{*}\right) .
$$

The first inequality is by monotonicity, the second by the definition of the supergradient, and the third from the KKT conditions as noted above. After rearranging we obtain $\frac{w_{i} v_{i}\left(y_{i}^{\prime}\right)}{v_{i}\left(y_{i}^{*}\right)} \leq$ $w_{i}+p^{\top}\left(\bar{y}_{i}-y_{i}^{*}\right)$. Summing the previous inequality for each agent $i \in \mathcal{A}^{\prime \prime}$ for a subset $\mathcal{A}^{\prime \prime} \subseteq \mathcal{A}^{\prime}$, and by definition of $\bar{y}_{i}$, we have

$$
\sum_{i \in \mathcal{A}^{\prime \prime}} \frac{w_{i} v_{i}\left(y_{i}^{\prime}\right)}{v_{i}\left(y_{i}^{*}\right)} \leq \sum_{i \in \mathcal{A}^{\prime \prime}} w_{i}+\sum_{i \in \mathcal{A}^{\prime \prime}} p^{\top}\left(\bar{y}_{i}-y_{i}^{*}\right) \leq \sum_{i \in \mathcal{A}^{\prime \prime}} w_{i}+p^{\top} \mathbb{1} .
$$

Since $p_{j}>0$ implies $\sum_{i \in \mathcal{A}^{\prime}} y_{i j}^{*}=1$ we have that $p^{\top} \mathbb{1}=p^{\top} \sum_{i \in \mathcal{A}^{\prime}} y_{i}^{*}$. Then, by Claim 4.3 we have

$$
\sum_{i \in \mathcal{A}^{\prime \prime}} w_{i} \frac{v_{i}\left(y_{i}^{\prime}\right)}{v_{i}\left(y_{i}^{*}\right)} \leq \sum_{i \in \mathcal{A}^{\prime \prime}} w_{i}+\sum_{i \in \mathcal{A}^{\prime}} w_{i}
$$

\subsection{The approximation guarantee for the mixed matching relaxation}

Lemma 3.4. Let $\mathcal{H} \subseteq \mathcal{G}$ with $|\mathcal{H}|=|\mathcal{A}|$. Let $\alpha>0$ and $y^{*}$ be an optimal and $y$ a feasible solution of (EG) such that $v_{i}\left(y_{i}\right) \geq \frac{1}{\alpha} v_{i}\left(y_{i}^{*}\right)$ for all $i \in \mathcal{A}^{\prime}$. Let $\pi$ be maximum weight assignment in the bipartite graph with colour classes $\mathcal{A}$ and $\mathcal{H}$, and edge weights $\omega_{i j}=w_{i} \log \left(v_{i}\left(y_{i}\right)+v_{i j}\right)$ for $i \in \mathcal{A}$, $j \in \mathcal{H}$. Then,

$$
\overline{\mathrm{NSW}}(y, \pi) \geq \frac{1}{2 \alpha} \overline{\mathrm{OPT}}_{\mathcal{H}} .
$$


Proof. Let $\pi^{*}$ be a maximum weight matching in the bipartite graph with colour classes $\mathcal{A}$ and $\mathcal{H}$ and with edge weights $q_{i}^{*}=w_{i} \log \left(v_{i}\left(y^{*}\right)+v_{i j}\right)$. Equivalently, $\pi^{*}$ is a matching maximizing

$$
\left(\prod_{i \in \mathcal{A}^{\prime}}\left(v_{i}\left(y_{i}^{*}\right)+v_{i \pi^{*}(i)}\right)^{w_{i}}\right)^{1 / \sum_{i \in \mathcal{A}} w_{i}} .
$$

We have the bounds

$$
\overline{\mathrm{NSW}}(y, \pi) \geq \overline{\mathrm{NSW}}\left(y, \pi^{*}\right) \geq \frac{1}{\alpha} \overline{\mathrm{NSW}}\left(y^{*}, \pi^{*}\right) .
$$

The first inequality is by the definition of $\pi$ as the maximum weight matching. The second inequality follows from the assumption $v_{i}\left(y_{i}\right) \geq \frac{1}{\alpha} v_{i}\left(y_{i}^{*}\right)$ for each $i \in \mathcal{A}^{\prime}$.

The rest of the proof is devoted to proving that $\overline{\mathrm{NSW}}\left(y^{*}, \pi^{*}\right) \geq \frac{1}{2} \overline{\mathrm{OPT}}_{\mathcal{H}}$; together with (4), this implies the statement. Let us introduce some notation. For an agent $i \in \mathcal{A}$, let $Y_{i}^{*}=v_{i}\left(y_{i}^{*}\right)$ be the value agent $i$ gets from the optimal fractional bundle $y^{*}$. Then,

$$
\overline{\operatorname{NSW}}\left(y^{*}, \pi^{*}\right)=\left(\prod_{i \in \mathcal{A}^{\prime}}\left(Y_{i}^{*}+v_{i \pi^{*}(i)}\right)^{w_{i}} \prod_{i \in A \backslash \mathcal{A}^{\prime}} v_{i \pi^{*}(i)}^{w_{i}}\right)^{1 / \sum_{i \in \mathcal{A}} w_{i}} .
$$

Let $\left(y^{\prime}, \varrho\right)$ be an optimal solution achieving $\overline{\mathrm{OPT}}_{\mathcal{H}}$. For an agent $i \in \mathcal{A}$ let $Y_{i}=v_{i}\left(y_{i}^{\prime}\right)$ be the value agent $i$ gets from the fractional allocation $y^{\prime}$. Then $\overline{\mathrm{OPT}}_{\mathcal{H}}=\overline{\mathrm{NSW}}\left(y^{\prime}, \varrho\right)=$ $\left(\prod_{i \in \mathcal{A}}\left(Y_{i}+v_{i \varrho(i)}\right)^{w_{i}}\right)^{1 / \sum_{i \in \mathcal{A}} w_{i}}$. By definition of the set $\mathcal{A}^{\prime}$, the agents in $\mathcal{A} \backslash \mathcal{A}^{\prime}$ do not value the items in $\mathcal{L}$. Thus, by monotonicity

$$
\overline{\mathrm{NSW}}\left(y^{\prime}, \varrho\right)=\left(\prod_{i \in \mathcal{A}^{\prime}}\left(Y_{i}+v_{i \varrho(i)}\right)^{w_{i}} \prod_{i \in \mathcal{A} \backslash \mathcal{A}^{\prime}} v_{i \varrho(i)}^{w_{i}}\right)^{1 / \sum_{i \in \mathcal{A}} w_{i}} .
$$

By the choice of $\pi^{*}$, we have

$$
\overline{\mathrm{NSW}}\left(y^{*}, \pi^{*}\right) \geq \overline{\mathrm{NSW}}\left(y^{*}, \varrho\right)=\left(\prod_{i \in \mathcal{A}^{\prime}}\left(Y_{i}^{*}+v_{i \varrho(i)}\right)^{w_{i}} \prod_{i \in \mathcal{A} \backslash \mathcal{A}^{\prime}} v_{i \varrho(i)}^{w_{i}}\right)^{1 / \sum_{i \in \mathcal{A}} w_{i}}
$$

Combining the last two we have: $\frac{\overline{\mathrm{NSW}}\left(y^{\prime}, \varrho\right)}{\overline{\mathrm{NSW}}\left(y^{*}, \pi^{*}\right)} \leq\left(\prod_{i \in \mathcal{A}^{\prime}}\left(\frac{Y_{i}+v_{i \varrho(i)}}{Y_{i}^{*}+v_{i \varrho(i)}}\right)^{w_{i}}\right)^{1 / \sum_{i \in \mathcal{A}} w_{i}}$.

Let $\mathcal{A}^{\prime \prime}=\left\{i \in \mathcal{A}^{\prime}: Y_{i}>Y_{i}^{*}\right\}$ be the set of agents that get more value from $y^{\prime}$ than $y^{*}$. Then, for $i \in \mathcal{A}^{\prime} \backslash \mathcal{A}^{\prime \prime}$ the fraction $\frac{Y_{i}+v_{i \varrho(i)}}{Y_{i}^{*}+v_{i \varrho(i)}}$ is trivially bounded by 1 . On the other hand, for $i \in \mathcal{A}^{\prime \prime}$ we have $\frac{Y_{i}+v_{i \varrho(i)}}{Y_{i}^{*}+v_{i \varrho(i)}} \leq \frac{Y_{i}}{Y_{i}^{*}}$. Since $\overline{\mathrm{OPT}}_{\mathcal{H}}=\overline{\mathrm{NSW}}\left(y^{\prime}, \varrho\right)$ it follows

$$
\frac{\overline{\mathrm{OPT}}_{\mathcal{H}}}{\overline{\mathrm{NSW}}\left(y^{*}, \pi^{*}\right)} \leq\left(\prod_{i \in \mathcal{A}^{\prime}}\left(\frac{Y_{i}+v_{i \varrho(i)}}{Y_{i}^{*}+v_{i \varrho(i)}}\right)^{w_{i}}\right)^{1 / \sum_{i \in \mathcal{A}} w_{i}} \leq\left(\prod_{i \in \mathcal{A}^{\prime \prime}}\left(\frac{Y_{i}}{Y_{i}^{*}}\right)^{w_{i}}\right)^{1 / \sum_{i \in A} w_{i}}
$$

We claim that the last expression is bounded by 2. By Lemma 4.1 we have $\sum_{i \in \mathcal{A}^{\prime \prime}} w_{i} \frac{Y_{i}}{Y_{i}^{*}} \leq$ $\sum_{i \in \mathcal{A}^{\prime \prime}} w_{i}+\sum_{i \in \mathcal{A}^{\prime}} w_{i}$. Then by the inequality between weighted arithmetic and geometric mean we have 


$$
\prod_{i \in \mathcal{A}^{\prime \prime}}\left(\frac{Y_{i}}{Y_{i}^{*}}\right)^{w_{i} / \sum_{i \in \mathcal{A}} w_{i}} \leq \frac{\sum_{i \in \mathcal{A}^{\prime \prime}} w_{i} \frac{Y_{i}}{Y_{i}^{*}}+\sum_{i \in \mathcal{A} \backslash \mathcal{A}^{\prime \prime}} 1}{\sum_{i \in \mathcal{A}} w_{i}} \leq \frac{\sum_{i \in \mathcal{A}^{\prime \prime}} w_{i}+\sum_{i \in \mathcal{A}^{\prime}} w_{i}+\left|\mathcal{A} \backslash \mathcal{A}^{\prime \prime}\right|}{\sum_{i \in \mathcal{A}} w_{i}} \leq 2 .
$$

The lemma follows.

\section{Phase IV: Obtaining a sparse approximate solution}

Recall that a continuous Rado valuation is defined as an optimum of the LP (2). For the valuation $v_{i}$ of agent $i \in \mathcal{A}$, this is defined by a bipartite graph $\left(\mathcal{G}, V_{i} ; E_{i}\right)$ with $\operatorname{costs}$ on the edges $c_{i}: E_{i} \rightarrow \mathbb{R}_{+}$, and a matroid $\mathcal{M}_{i}=\left(V_{i}, \mathcal{I}_{i}\right)$ with a rank function $r_{i}=r_{\mathcal{M}_{i}}$.

The program (EG) for $\mathcal{A}^{\prime}$ and $\mathcal{L}=\mathcal{G} \backslash \mathcal{H}$ can be thus written as follows.

$$
\begin{array}{ll}
\max & \sum_{i \in \mathcal{A}^{\prime}} w_{i} \log \left(\sum_{j \in \mathcal{L}} \sum_{k \in V_{i}} c_{i j k} z_{i j k}\right) \\
\text { s.t.: } & \sum_{i \in \mathcal{A}^{\prime}} y_{i j} \leq 1 \quad \forall j \in \mathcal{L} \\
\sum_{k \in V_{i}} z_{i j k} \leq y_{i j} \quad \forall i \in \mathcal{A}^{\prime}, \forall j \in \mathcal{L} \\
\sum_{j \in \mathcal{L}} \sum_{k \in S} z_{i j k} \leq r_{i}(S) \quad \forall i \in \mathcal{A}^{\prime}, \forall S \subseteq V_{i} \\
y \geq 0, \quad z \geq 0 .
\end{array}
$$

Without loss of generality we can assume that the second set of constraints always holds with equality, i.e., $y_{i j}=\sum_{k \in V_{i}} z_{i j k}$ for $j \in \mathcal{L}$ and $i \in \mathcal{A}^{\prime}$. By eliminating the variables $y$, the program (EG) becomes:

$$
\begin{array}{cc}
\max & \sum_{i \in \mathcal{A}^{\prime}} w_{i} \log \left(\sum_{j \in \mathcal{L}} \sum_{k \in V_{i}} c_{i j k} z_{i j k}\right) \\
\text { s.t.: } & \sum_{i \in \mathcal{A}^{\prime}} \sum_{k \in V_{i}} z_{i j k} \leq 1 \quad \forall j \in \mathcal{L} \\
& \sum_{j \in \mathcal{L}} \sum_{k \in S} z_{i j k} \leq r_{i}(S) \quad \forall i \in \mathcal{A}^{\prime}, \forall S \subseteq V_{i} \\
z \geq 0, &
\end{array}
$$

Using this formulation, we first show that the Eisenberg-Gale type convex program (EG) can be solved exactly in polynomial time for Rado valuations (Section 5.1). We then transform the optimal solution to a sparse approximate solution (Section 5.2).

\subsection{Solving the Eisenberg-Gale relaxation}

In this section, we prove the following lemma.

Lemma 3.7. Suppose that for each agent $i \in \mathcal{A}, v_{i}$ is a Rado valuation given by a bipartite $\operatorname{graph}\left(\mathcal{G}, V_{i} ; E_{i}\right)$, integer costs $c_{i}: E_{i} \rightarrow \mathbb{Z}$ and a matroid $\mathcal{M}_{i}=\left(V_{i}, \mathcal{I}_{i}\right)$ as in Definition 2.4. Let $T=\max _{i \in \mathcal{A}}\left|V_{i}\right|$, and $C=\max _{i \in \mathcal{A}}\left\|c_{i}\right\|_{\infty}$. Let the weights $w_{i}>0$ be rational numbers given as quotients of two integers at most $U$. Assume the matroids $\mathcal{M}_{i}$ are given by rank oracles. Then, 
(EG) has a rational solution with poly $(|\mathcal{A}|,|\mathcal{G}|, T, \log C, \log U)$ bit-complexity, and such a solution can be found in $\operatorname{poly}(|\mathcal{A}|,|\mathcal{G}|, T, \log C, \log U)$ arithmetic operations and calls to the matroid rank oracles.

As noted above, (EG) with Rado valuations for the set of agents $\mathcal{A}^{\prime}$ and set of goods $\mathcal{L}$ is equivalent to (EG-Rado). Throughout, we assume this program is feasible, i.e. it has a solution with finite objective value. This is a mild condition only requiring the existence of at least one edge $(j, k) \in E_{i}$ with $c_{i j k}>0$ and $r_{i}(\{k\})=1$ for every $i \in \mathcal{A}^{\prime}$.

In general, one can only expect to solve convex programs approximately: no rational solution may even exist. Vazirani [69] defines rational convex programs where a finite optimum exists with bounded bit-complexity in the input size, where the input is described by a finite set of parameters. This model is not directly applicable for our program (EG-Rado) as it is described with an exponential number of constraints. The bound poly $(|\mathcal{A}|,|\mathcal{G}|, T, \log C, \log U)$ does not take into account the matroidal constraints; it is polynomial in the amount of information needed to describe the objective function. ${ }^{7}$

We first show that the set of optimal solutions is a polytope where the vertices have polynomially bounded bit-complexity.

Lemma 5.1. For an NSW problem instance with Rado valuations as in Lemma 3.7, the set of optimal solutions forms a polytope. The bit-complexity of each vertex of this polytope is bounded as $\operatorname{poly}(|\mathcal{A}|,|\mathcal{G}|, T, \log C, \log U)$.

To prove the above lemma we use the KKT conditions for (EG-Rado). Let $p_{j}$ 's and $\alpha_{i}(S)$ 's denote the Lagrange multipliers corresponding to the first and second sets of the constraints, respectively. It holds:

(i) $\forall j \in \mathcal{L}: p_{j} \geq 0$.

(ii) $\forall i \in \mathcal{A}^{\prime}, \forall S \subseteq V_{i}: \alpha_{i}(S) \geq 0$.

(iii) $\forall j \in \mathcal{L}: p_{j}>0 \Longrightarrow \sum_{i \in \mathcal{A}^{\prime}, k \in V_{i}} z_{i j k}=1$.

(iv) $\forall i \in \mathcal{A}^{\prime}, \forall S \subseteq V_{i}: \alpha_{i}(S)>0 \Longrightarrow \sum_{j \in \mathcal{L}, k \in S} z_{i j k}=r_{i}(S)$.

(v) $\forall i \in \mathcal{A}^{\prime}, \forall j \in \mathcal{L}, \forall k^{\prime} \in V_{i}: \frac{c_{i j k}}{p_{j}+\sum_{S: k \in S} \alpha_{i}(S)} \leq \frac{\sum_{j \in \mathcal{L}, k^{\prime} \in V_{i}} c_{i j k^{\prime}} z_{i j k^{\prime}}}{w_{i}}$.

(vi) $\forall i \in \mathcal{A}^{\prime}, \forall j \in \mathcal{L}: z_{i j k}>0 \Longrightarrow \frac{c_{i j k}}{p_{j}+\sum_{S: k \in S} \alpha_{i}(S)}=\frac{\sum_{j \in \mathcal{L}, k \in V_{i}} c_{i j k^{\prime}} z_{i j k^{\prime}}}{w_{i}}$.

In $(v)$ and $(v i)$, we have divided the conditions by $p_{j}+\sum_{S: k \in S} \alpha_{i}(S)$ and multiplied by $\sum_{j \in \mathcal{L}, k^{\prime} \in V_{i}} c_{i j k^{\prime}} z_{i j k^{\prime}}$. By the feasibility assumption, both these must be positive.

We say that $(p, \alpha)$ are optimal Lagrange multipliers if they satisfy $(i)-(v i)$ together with any optimal solution $z$ to (EG-Rado).

\footnotetext{
${ }^{7}$ We note that for exponential size linear programs, a standard way to bound the encoding size is giving bounds on facet/vertex-complexity, defined later in this section. The program (EG-Rado) maximizes a concave function over a polytope that has facet complexity $O(|\mathcal{A}| T)$.
} 
Claim 5.2. There exists an optimal solution $z$ with optimal Lagrange multipliers $(p, \alpha)$ with the following property: for every agent $i \in \mathcal{A}^{\prime}$, the support of the vector $\alpha_{i}$ is a chain of sets $S_{1}^{(i)} \subset$ $\cdots \subset S_{h_{i}}^{(i)} \subseteq V_{i}$ for some $h_{i} \in \mathbb{N}$.

Proof of Claim. We use a standard uncrossing argument. Let $z$ be an optimal solution to (EG-Rado). Let us consider the set of optimal Lagrange multipliers $(p, \alpha)$. For a fixed $z$, the set of vectors $(p, \alpha)$ satisfying the constraints $(i)-(v i)$ forms a polytope, since each constraint can be equivalently written as a linear constraint, and (iii), $(i v)$, and (vi) imply boundedness. Thus, there exists a solution $(p, \alpha)$ that maximizes the objective

$$
\varphi(p, \alpha):=\sum_{i \in \mathcal{A}^{\prime}} \sum_{S \subseteq V_{i}}|S|^{2} \alpha_{i}(S)
$$

We claim that such a solution satisfies the conditions. This follows by showing that for each $i \in \mathcal{A}^{\prime}$, if $\alpha_{i}(X), \alpha_{i}(Y)>0$ then either $X \subseteq Y$ or $Y \subseteq X$.

For a contradiction, assume $X \backslash Y, Y \backslash X \neq \emptyset$, and let $\varepsilon:=\min \left\{\alpha_{i}(X), \alpha_{i}(Y)\right\}>0$. Let us define $\alpha^{\prime}$ as follows:

- $\alpha_{i}^{\prime}(X \cup Y)=\alpha_{i}(X \cup Y)+\varepsilon$

- $\alpha^{\prime}(X)=\alpha(X)-\varepsilon$ and $\alpha^{\prime}(Y)=\alpha(Y)-\varepsilon$;

- if $X \cap Y \neq \emptyset$, then $\alpha_{i}^{\prime}(X \cap Y)=\alpha_{i}(X \cap Y)+\varepsilon$;

- if $S \notin\{X, Y, X \cup Y, X \cap Y\}$ then $\alpha_{i}^{\prime}(S)=\alpha_{i}(S)$; and

- if $j \neq i$ then $\alpha_{j}^{\prime}(S)=\alpha_{j}(S)$ for all $S$.

We claim that $\left(p, \alpha^{\prime}\right)$ are also optimal Lagrange multipliers. This gives a contradiction, since $\varphi\left(p, \alpha^{\prime}\right)>\varphi(p, \alpha)$. Constraints $(i)-(i i i)$ are immediate. Constraints $(v)$ and $(v i)$ follow since $\sum_{S: k \in S} \alpha_{i}^{\prime}(S)=\sum_{S: k \in S} \alpha_{i}(S)$ holds for all $i \in \mathcal{A}^{\prime}$ and all $k \in V_{i}$. Finally, (iv) follows by observing that for any $i \in \mathcal{A}^{\prime}$ and any $j \in \mathcal{L}$,

$$
\begin{aligned}
& \sum_{j \in \mathcal{L}, k \in X} z_{i j k}+\sum_{j \in \mathcal{L}, k \in Y} z_{i j k}=r_{i}(X)+r_{i}(Y) \geq r_{i}(X \cup Y)+r_{i}(X \cap Y) \\
\geq & \sum_{j \in \mathcal{L}, k \in X \cap Y} z_{i j k}+\sum_{j \in \mathcal{L}, k \in X \cup Y} z_{i j k}=\sum_{j \in \mathcal{L}, k \in X} z_{i j k}+\sum_{j \in \mathcal{L}, k \in Y} z_{i j k},
\end{aligned}
$$

using the submodularity of $r_{i}$. We must have equality throughout, implying (iv) for $S=X \cup Y$ and $S=X \cap Y$.

Proof of Lemma 5.1. Let $z$ be any optimal solution to (EG-Rado) and let $(p, \alpha)$ be any optimal Lagrange multipliers as in Claim 5.2, with $\alpha_{i}$ supported on the chain $S_{1}^{(i)} \subset S_{2}^{(i)} \subset \ldots \subset S_{h_{i}}^{(i)}$.

Let $\mathcal{L}^{\prime} \subseteq \mathcal{L}$ be the subset of goods with $p_{j}>0$, and let $E_{i}^{\prime} \subseteq E_{i}$ be the set of edges $(j, k)$ for which $c_{i j k} /\left(p_{j}+\sum_{S: k \in S} \alpha_{i}(S)\right)$ is maximized. Clearly, $z_{i j k}>0$ only if $(j, k) \in E_{i}^{\prime}$.

We perform the following variable substitution:

$$
q_{j}:=\frac{1}{p_{j}} \quad \forall j \in \mathcal{L}, \quad \text { and } \quad Q_{j t}^{(i)}:=\frac{1}{p_{j}+\sum_{b=t}^{h_{i}} \alpha_{i}\left(S_{b}^{(i)}\right)} \quad \forall i \in \mathcal{A}^{\prime}, \forall t \in\left[h_{i}\right] .
$$


We show that, provided the supports $\mathcal{L}^{\prime}, E_{i}^{\prime}$, we can define a linear program in the variables $q_{j}$ 's, $Q_{j t}^{(i)}$ 's, and $z_{i j k}$ as follows. We include all feasibility constraints on $z_{i j k}$ from (EG-Rado) and the following additional constraints:

$$
\begin{aligned}
\sum_{i \in \mathcal{A}^{\prime}, k \in V_{i}} z_{i j k} & =1 & & \forall j \in \mathcal{L}^{\prime} \\
\sum_{j \in \mathcal{L}, k \in S} z_{i j k} & =r_{i}(S) & & \forall i \in A^{\prime}, \forall S \subseteq V_{i} \\
w_{i} c_{i j k} Q_{j t}^{(i)} & \leq \sum_{j \in \mathcal{L}, k^{\prime} \in V_{i}} c_{i j k^{\prime}} z_{i j k^{\prime}} & & \forall i \in \mathcal{A}^{\prime}, \forall(j, k) \in E_{i} \text {, and } t \text { s.t. } k \in S_{t}^{(i)} \backslash S_{t-1}^{(i)} \\
w_{i} c_{i j k} Q_{j t}^{(i)} & =\sum_{j \in \mathcal{L}, k^{\prime} \in V_{i}} c_{i j k^{\prime}} z_{i j k^{\prime}} & & \forall i \in \mathcal{A}^{\prime}, \forall(j, k) \in E_{i}^{\prime}, \text { and } t \text { s.t. } k \in S_{t}^{(i)} \backslash S_{t-1}^{(i)} \\
Q_{j t}^{(i)} & \leq Q_{j(t+1)}^{(i)} & & \forall i \in \mathcal{A}^{\prime}, j \in \mathcal{L}^{\prime}, t \in\left[h_{i}-1\right] \\
q_{j} & =0 & & \forall j \in \mathcal{L} \backslash \mathcal{L}^{\prime} \\
z_{i j k} & =0 & & \forall i \in \mathcal{A}^{\prime},(j, k) \in E_{i} \backslash E_{i}^{\prime} \\
Q, q & \geq 0 & &
\end{aligned}
$$

Let $P \in \mathbb{R}^{\left(\sum_{i \in \mathcal{A}^{\prime}}\left|E_{i}\right|\right) \times \mathcal{L}^{\prime} \times\left(\sum_{j \in F^{\prime}} h_{i}\right)}$ be the set of feasible solutions to this LP. According to $(i)-(v i)$, $(z, q, Q) \in P$, where $(q, Q)$ is obtained from $(p, \alpha)$ as in (5). Conversely, if $\left(z^{\prime}, q^{\prime}, Q^{\prime}\right) \in P$, then we can map $\left(q^{\prime}, Q^{\prime}\right)$ to a nonnegative $\left(p^{\prime}, \alpha^{\prime}\right)$ such that (5) holds and $\left(z^{\prime}, p^{\prime}, \alpha^{\prime}\right)$ satisfy $(i)-(v i)$.

Since all coefficients in the system are rational numbers from the input, and the feasible region $P$ is bounded, it follows that $P$ is a polytope where all basic feasible solutions are rational vectors with encoding size polynomially bounded in the input.

Let us fix $\left(q^{\prime}, Q^{\prime}\right)$ in a basic feasible solution, and let $P^{\prime \prime}=\left\{z^{\prime \prime}:\left(z^{\prime \prime}, q^{\prime}, Q^{\prime}\right) \in P\right\}$. Then, $z^{\prime \prime} \in P^{\prime \prime}$ if and only if $z^{\prime \prime}$ is optimal with respect to (EG-Rado). Further, $P^{\prime \prime}$ is a polytope defined by linear constraints with polynomially bounded coefficients. Thus, the claim follows.

The Ellipsoid Method for Rational Polyhedra We quickly recall some relevant concepts for the Ellipsoid Method from the book [32] by Grötschel, Lovász, and Schrijver. A strong separation oracle for the convex set $K \subseteq \mathbb{R}^{n}$ takes as input a vector $x \in \mathbb{R}^{n}$, and either returns the answer $x \in K$, or returns a vector $a \in \mathbb{R}^{n}$ such that $\langle a, x\rangle>\max \{\langle a, z\rangle: z \in K\}$.

Let us recall the definitions of facet and vertex complexity. We only include the definitions for polytopes, instead of general polyhedra.

Definition 5.3 ([32, Definition (6.2.2)]). Let $P \subseteq \mathbb{R}^{n}$ be a polytope.

1. We say that $P$ has facet-complexity at most $\varphi$, if $P$ can be defined by a system of linear inequalities with rational coefficients such that each inequality has encoding length at most $\varphi$. If $P=\mathbb{R}^{n}$, we require $\varphi \geq n+1$. The triple $(P ; n, \varphi)$ is called a well-described polytope.

2. We say that $P$ has vertex-complexity at most $\nu$, if $P$ is the convex hull of a finite set of rational vectors, all having encoding length at most $\nu . P=\emptyset$, then we require $\nu \geq n$.

Lemma 5.4 ([32, Lemma (6.2.4)]). If $P$ has vertex-complexity at most $\nu$, then $P$ has facetcomplexity at most $3 n^{2} \nu$.

Theorem 5.5 ([32, Theorems (6.4.9), (6.5.7)]). For a well-described polyhedron $(P ; n, \varphi)$ given by a strong separation oracle, there exists oracle-polynomial time algorithm that either returns a vertex 
solution $x \in P$, or concludes that $P=\emptyset$. Given a linear objective function $\langle c, x\rangle$, if $P \neq \emptyset$ then there exists an oracle-polynomial time algorithm that finds an optimal vertex solution to $\max \langle c, x\rangle$ s.t. $x \in P$.

An oracle-polynomial time algorithm means that the number of arithmetic operations and calls to the strong separation oracle is bounded as $\operatorname{poly}(\varphi)$; note that $\varphi \geq n$.

Proof of Lemma 3.7. Let $P$ be the set of feasible solutions and $P^{*}$ the set of optimal solutions to (EG-Rado). We note that $P \neq \emptyset$ since $z=0$ is a feasible solution. Further, $P^{*} \neq \emptyset$ since $P$ is bounded. Lemma 5.1 asserts that this is a nonempty polytope with vertex-complexity $\operatorname{poly}(|\mathcal{A}|,|\mathcal{G}|, T, \log C, \log U)$; thus $\left(P^{*}, \sum_{i \in \mathcal{A}}\left|E_{i}\right|, \varphi\right)$ is a well-described polytope for some $\varphi \in$ $\operatorname{poly}(|\mathcal{A}|,|\mathcal{G}|, T, \log C, \log U)$ by Lemma 5.4 .

We now describe the strong separation oracle to $P^{*}$. For a vector $z \in \mathbb{R}^{\times_{i \in \mathcal{A}} E_{i}}$, we first check whether $z \in P$. Checking the first set of $|\mathcal{A}|$ constraints is straightforward. The submodular constraints can be verified by solving $|\mathcal{A}|$ submodular function minimization problems. We either conclude $z \in P$, or obtain a separating hyperplane for $z$ and $P$ that is also a separating hyperplane for $z$ and $P^{*}$.

If $z \in P$, the we compute the gradient $\nabla f(z)$, where $f(z)$ denotes the objective function. We then solve the linear optimization problem $\max \langle\nabla f(z), x\rangle$ s.t. $x \in P$. $\left(P^{*}, \sum_{i \in \mathcal{A}}\left|E_{i}\right|, \sum_{i \in \mathcal{A}}\left|E_{i}\right|+\right.$ $\log T)$ is a well-described polytope since all coefficients are 0 and 1 and the left hand side values are at most $T$. Using the strong separation oracle for $P$ we just described, the second half of Theorem 5.5 shows that we can find an optimal solution $x^{*} \in P$ in time poly $(|\mathcal{A}|,|\mathcal{G}|, T, \log C, \log U)$.

If $\max \left\langle\nabla f(z), x^{*}\right\rangle=\max \langle\nabla f(z), z\rangle$, i.e., if $z$ itself is an optimal solution, then we conclude that $z \in P^{*}$. Otherwise, $\langle\nabla f(z), x\rangle>\langle\nabla f(z), z\rangle$ is a valid separating hyperplane.

Thus, by the first half of Theorem 5.5, we can find an optimal solution $x \in P^{*}$ in time $\operatorname{poly}(|\mathcal{A}|,|\mathcal{G}|, T, \log C, \log U)$.

This method requires the implementation of the ellipsoid method for linear optimization inside the separation oracle. We now show that this can be easily avoided by always using the hyperplane $\langle\nabla f(z), x\rangle>\langle\nabla f(z), z\rangle$, without solving the LP. If $z \in P \backslash P^{*}$, then this is always valid, but if $z \in P^{*}$, then this holds with equality instead of strict inequality.

Nevertheless, we can run the ellipsoid method using the gradients as separating directions (without solving the LP). This ultimately leads to concluding $P^{*}=\emptyset$, since the algorithm returns a separating hyperplane for every $z \in \mathbb{R}^{\times_{i \in \mathcal{A}} E_{i}}$. At this point, we consider the feasible solution $z \in P$ with the largest objective value $f(z)$ visited by the algorithm, and conclude that this solution must have been optimal. This is true since if no optimal solutions would have been visited, then every separating hyperplane we used would be a valid strong separator for $P^{*}$, and thus, we could not have reached the false conclusion $P^{*}=\emptyset$.

Remark 5.6. We note that a similar argument was used by Jain [37, Theorem 12], showing that whenever a convex set is given with a strong separation oracle and is guaranteed to contain a point of bit-complexity at most $\nu$, then a feasible solution can be found in polynomial time, using simultaneous Diophantine approximation. Our proof leverages the stronger property that the optimal solution set $P^{*}$ is a well-described polytope.

\subsection{Sparse solutions to Eisenberg-Gale relaxation}

In this section we prove Lemma 3.8. Recall that the polytope $P^{*}$ is the set of optimal solutions to (EG-Rado) as in Lemma 5.1. In Lemma 5.7 and Corollary 5.8, we show that the solution of every vertex solution of $P^{*}$ is sparse. In Lemma 3.8 we further sparsify such a solution by losing at 
most half of the value for each agent. The arguments in both steps rely on bounding the number of non-zero variables in particular linear systems.

Consider an optimal solution $z$ for (EG-Rado) that is also a basic solution to $P^{*}$. According to Theorem 5.5, we can require that the optimal solution found in Lemma 3.7 is a basic solution. We define $v_{i}^{*}:=\sum_{k \in V_{i}} c_{i j k} z_{i j k}$ as the optimum utility value attained by agent $i \in \mathcal{A}^{\prime}$; by strict convexity of the objective, these values are the same for all optimal solutions.

Lemma 5.7. Every optimal solution $z \in P^{*}$ satisfies $\left|\operatorname{supp}\left(z^{\prime}\right)\right| \leq\left|\mathcal{A}^{\prime}\right|+2\left|\mathcal{L}^{+}\left(z^{\prime}\right)\right|-\left|R_{1}\right|-\left|R_{2}\right|$, where

$$
\begin{aligned}
\mathcal{L}^{+}(z) & =\left\{j \in \mathcal{L}: \sum_{i \in \mathcal{A}^{\prime}} \sum_{k \in V_{i}} z_{i j k}>0\right\}, \\
R_{1} & =\left\{j \in \mathcal{L}: \exists ! i \in \mathcal{A}^{\prime} \text { such that } 0<\sum_{k \in V_{i}} z_{i j k}<1\right\}, \\
R_{2} & =\left\{j \in \mathcal{L}: \exists ! i \in \mathcal{A}^{\prime} \text { such that } z_{i j k}=1 \text { for some } k \in V_{i}\right\} .
\end{aligned}
$$

The set $\mathcal{L}^{+}$is the set of allocated items in $\mathcal{L}$ by $z ; R_{1}$ is the set of items in $\mathcal{L}$ each of which is allocated to one agent only, but the item is not fully allocated; and $R_{2}$ is the set of items in $\mathcal{L}$ each of which is fully allocated to agent via single edge of the graph $\left(\mathcal{G}, V_{i} ; E_{i}\right)$. Obviously, $R_{1}$ and $R_{2}$ are disjoint.

Proof of Lemma 5.7. The following LP gives a description of $P^{*}$. We note that this is a different description from the extended system in the proof of Lemma 5.1: here, we can make use of the optimal values $v_{i}^{*}$ and thus do not require the dual variables. Note that the notion of vertex solutions is independent of the describing system.

$$
\begin{aligned}
\sum_{j \in \mathcal{L}, k \in V_{i}} c_{i j k} z_{i j k} & \geq \hat{v}_{i}^{*} & & \forall i \in \mathcal{A}^{\prime} \\
\sum_{i \in \mathcal{A}^{\prime}, k \in V_{i}} z_{i j k} & \leq 1 & & \forall j \in \mathcal{L} \\
\sum_{j \in \mathcal{L}, k \in S} z_{i j k} & \leq r_{i}(S) & & \forall i \in \mathcal{A}^{\prime}, \forall S \subseteq V_{i} \\
z & \geq 0 . & &
\end{aligned}
$$

In order to prove the bound on the support of a vertex (basic feasible) solution to $P^{*}$, we upperbound the number of linearly independent tight constraints. Trivially, there are at most $\left|\mathcal{A}^{\prime}\right|$ tight constraints of the first type. By definition of sets $\mathcal{L}^{+}$and $R_{1}$ there are at most $\left|\mathcal{L}^{+}\right|-\left|R_{1}\right|$ tight constraints of the second type.

Let us bound the maximal number of tight submodular constraints. By Claim 5.2, for each agent $i \in \mathcal{A}^{\prime}$, the maximal set of linearly independent tight submodular constraints forms a chain. Formally, for $i \in \mathcal{A}^{\prime}$ there exist sets $S_{1}^{i} \subset S_{2}^{i} \subset \cdots \subset S_{h_{i}}^{i} \subseteq V_{i}$, such that the set of constraints $\left\{\sum_{j \in \mathcal{G}, k \in S_{t}^{i}} z_{i j k} \leq r_{i}\left(S_{t}^{i}\right)\right\}_{t=1}^{h_{i}}$ generates all the tight submodular constraints for agent $i$. All together, there are at most $\left|\mathcal{A}^{\prime}\right|+\left|\mathcal{L}^{+}\right|-\left|R_{1}\right|+\sum_{i \in \mathcal{A}^{\prime}} h_{i}$ tight constraints.

Now, let us consider an element $j \in R_{2}$ and let $i$ be the agent such that $z_{i j k}=1$ for some $k \in V_{i}$. Since $r_{i}$ is rank function we have $z_{i j k}=1=r_{i}(\{k\})$. Let $S_{b}^{i}$ be the smallest set in the $i$-th chain containing $k$. Since $\{k\}$ is also tight we can assume that $k=S_{b}^{i} \backslash S_{b-1}^{i}$. Therefore, the tight inequalities corresponding to $S_{b}^{i}, S_{b-1}^{i}$ and $z_{i j k} \leq 1$ (or equivalently $\sum_{k \in V_{i}} z_{i j k} \leq 1$ ) are not linearly independent and we can drop the inequality corresponding to $z_{i j k} \leq 1$ from the minimal set 
of linearly independent tight inequalities. In other words, we do not have to count the inequality corresponding to $j$, for $j \in R_{2}$ and we can replace the term $\left|\mathcal{L}_{+}\right|$by $\left|\mathcal{L}_{+}\right|-\left|R_{2}\right|$.

Further, by flow conservation we have $\left|\mathcal{L}^{+}\right| \geq \sum_{i \in \mathcal{A}^{\prime}, j \in \mathcal{L}, k \in V_{i}} z_{i j k} \geq \sum_{i \in \mathcal{A}^{\prime}} r_{i}\left(S_{h_{i}}^{i}\right) \geq \sum_{i \in \mathcal{A}^{\prime}} h_{i}$. Thus,

$$
|\operatorname{supp}(z)| \leq\left|\mathcal{A}^{\prime}\right|+2\left|\mathcal{L}^{+}\right|-\left|R_{1}\right|-\left|R_{2}\right|
$$

Corollary 5.8. Consider an optimal vertex solution $y$ of (EG) for Rado valuations. Then, $|\operatorname{supp}(y)| \leq\left|\mathcal{A}^{\prime}\right|+2\left|\mathcal{L}^{+}(y)\right|-\left|\mathcal{L}_{1}(y)\right|$, where

$$
\begin{aligned}
& \mathcal{L}^{+}(y)=\left\{j \in \mathcal{L}: \sum_{i \in \mathcal{A}^{\prime}} y_{i j}>0\right\}, \\
& \mathcal{L}_{1}(y)=\left\{j \in \mathcal{L}: \exists ! i \in \mathcal{A}^{\prime} \text { such that } y_{i j}>0\right\} .
\end{aligned}
$$

Proof. The optimal vertex solution $y$ can be written as $y_{i j}=\sum_{(i, k) \in E_{i}} z_{i j k}$ for a vertex solution $z$ of $P^{*}$. We have $|\operatorname{supp}(z)| \leq\left|\mathcal{A}^{\prime}\right|+2\left|\mathcal{L}^{+}\right|-\left|R_{1}\right|-\left|R_{2}\right|$. The first condition holds by definition of $y$. By construction we also have $\mathcal{L}^{+}(y)=\mathcal{L}^{+}(z)=$ : $\mathcal{L}^{+}$. Moreover, $R_{1}, R_{2} \subseteq \mathcal{L}_{1}$.

By definition of $\mathcal{L}_{1}, R_{1}$ and $R_{2}$; we have $j \in \mathcal{L}_{1} \backslash\left(R_{1} \cup R_{2}\right)$ if and only if $j$ is allocated fully to a unique agent $i$ and there exist different $k_{1}, k_{2} \in V_{i}$ with $z_{i j k_{1}}>0$ and $z_{i j k_{2}}>0$. Both variables $z_{i j k_{1}}$ and $z_{i j k_{2}}$ contribute that $y_{i j}>0$ for the same $i, j$. Thus,

$$
|\operatorname{supp}(y)| \leq\left|\mathcal{A}^{\prime}\right|+2\left|\mathcal{L}^{+}\right|-\left|R_{1}\right|-\left|R_{2}\right|-\left|\mathcal{L}_{1} \backslash\left(R_{1} \cup R_{2}\right)\right|=\left|\mathcal{A}^{\prime}\right|+2\left|\mathcal{L}^{+}\right|-\left|\mathcal{L}_{1}\right| .
$$

Further sparsification We showed that any basic optimal solution to (EG) under Rado valuations has support of size $\left|\mathcal{A}^{\prime}\right|+2\left|\mathcal{L}_{+}\right|-\left|\mathcal{L}_{1}\right|$. Next, we show that any such sparse solution can be further sparsified by losing a fraction of valuation of each agent. The main observation is that given a feasible allocation for a Rado valuation function, all "sub-allocations" behave in a "locally subadditive" way, as explained next.

Let $y^{\prime}$ be a feasible allocation and $z^{\prime}$ its corresponding representation in (EG-Rado). Our argument will scale down $y_{i j}=q_{i j} y_{i j}^{\prime}$ for some $q_{i j} \in[0,1]$. We have $v_{i}\left(y_{i}^{\prime}\right)=\sum_{j \in \mathcal{L}, k \in V_{i}} c_{i j k} z_{i j k}^{\prime}$. Therefore, we can write $v_{i}\left(y_{i}^{\prime}\right)=\sum_{j \in \mathcal{L}} u(i, j)$ where $u(i, j)=\sum_{k \in V_{i}} c_{i j k} z_{i j k}^{\prime}$ is the value agent $i$ gets from good $j$. Hence, we can represent $y_{i j}=q_{i j} y_{i j}^{\prime}$ as $y_{i j}=q_{i j} \sum_{k \in V_{i}} z_{i j k}^{\prime}$. Assuming $q_{i j} \in[0,1]$ we have

$$
v_{i}\left(y_{i}\right) \geq \sum_{j \in \mathcal{L}, k \in V_{i}} q_{i j} \cdot c_{i j k} z_{i j k}^{\prime}=\sum_{j \in \mathcal{L}} q_{i j} \cdot u(i, j),
$$

where we use the fact that whenever $z^{\prime}$ is feasible for (EG-Rado) then so is the allocation given by $q_{i j} z_{i j k}^{\prime}$ for $j \in \mathcal{L}, k \in V_{i}$. In particular, this justifies the notation $y_{i j}=q_{i j} y_{i j}^{\prime}$ for $q_{i j} \in[0,1]$ and it holds that $v_{i}\left(y_{i}\right) \geq \sum_{j \in \mathcal{L}} q_{i j} u(i, j)$. Such a property is used to prove the following lemma.

Lemma 3.8. Suppose the functions $v_{i}$ are Rado valuations, and let $\hat{y}$ be a feasible solution to (EG). Then, in polynomial time we can find a feasible solution y such that

(i) $v_{i}(y) \geq \frac{1}{2} v_{i}(\hat{y})$,

(ii) $|\operatorname{supp}(y)| \leq 2\left|\mathcal{A}^{\prime}\right|+\left|\mathcal{L}^{+}\right|$where $\mathcal{L}^{+}:=\mathcal{L}^{+}(y)=\left\{j \in \mathcal{G} \backslash \mathcal{H}: \sum_{i \in \mathcal{A}^{\prime}} y_{i j}>0\right\}$.

Given a $\hat{y}$, we can transform it to a vector $y^{\prime}$ with $\left|\operatorname{supp}\left(y^{\prime}\right)\right| \leq\left|\mathcal{A}^{\prime}\right|+2\left|\mathcal{L}^{+}\left(y^{\prime}\right)\right|-\left|\mathcal{L}_{1}\left(y^{\prime}\right)\right|$ by Corollary 5.8. Then, the idea is to exhibit $q$ such that the vector $y$ defined as $y_{i j}=q_{i j} y_{i j}^{\prime}$ satisfies the lemma. Such $q$ needs to preserve at least half of the value for each agent and should set at least $\left|\mathcal{L}^{+}\right|-\left|\mathcal{L}_{1}\right|-\left|\mathcal{A}^{\prime}\right|$ values of $y_{i j}^{\prime}$ to 0 . We can find such a $q$ as a basic feasible solution of a system of linear (in)equalities. 
Proof. Let $y^{\prime}$ be a solution of (EG) with $\left|\operatorname{supp}\left(y^{\prime}\right)\right| \leq\left|\mathcal{A}^{\prime}\right|+2\left|\mathcal{L}^{+}\left(y^{\prime}\right)\right|-\left|\mathcal{L}_{1}\left(y^{\prime}\right)\right|$, given by Corollary 5.8. Let $D=\left\{j \in \mathcal{L}^{+}\left(y^{\prime}\right): \exists i, i^{\prime}, i \neq i^{\prime}\right.$ such that $y_{i j}^{\prime}>0$ and $\left.y_{i^{\prime} j}^{\prime}>0\right\}$, i.e., $D$ is the set of items in $\mathcal{L}^{+}\left(y^{\prime}\right)$ allocated to at least two different agents by $y^{\prime}$. Hence, $|D|=\left|\mathcal{L}^{+}\left(y^{\prime}\right)\right|-\left|\mathcal{L}_{1}\left(y^{\prime}\right)\right|$. For each $j \in D$, let $D(j)$ be a set containing two different agents $i, i^{\prime}$ getting the item $j$ in $y^{\prime}$. Such two agents are picked arbitrarily, but fixed throughout the proof for each $j$. Let $\mathcal{A}^{\prime \prime}=\cup_{j \in D} D(j)$.

We consider the following linear system with variables $q$. The value $q_{i j}$ represents the fraction of $y_{i j}^{\prime}$ agent $i$ keeps. By the above, if agent obtained $u(i, j)$ value from $y_{i j}^{\prime}$ units of $j$ then agent receives $q_{i j} u(i, j)$ value from $q_{i j} y_{i j}^{\prime}$ units of good $j$ whenever $q_{i j} \in[0,1]$.

$$
\begin{aligned}
\sum_{j \in D} q_{i j} u(i, j) & \geq \frac{1}{2} \sum_{j \in D} u(i, j) & & \forall i \in \mathcal{A}^{\prime \prime} \\
q_{i j}+q_{i^{\prime} j} & =1 & & \\
q & \geq 0 . & & \forall j \in D,\left\{i, i^{\prime}\right\}=D(j)
\end{aligned}
$$

Let us define $y$ : set $y_{i j}=0$ if $q_{i j}=0$ and $y_{i j}=y_{i j}^{\prime}$ for all other values. Then for any feasible $q$ we have

- The second set of constraints together with non-negativity of $q$ guarantees $q_{i j} \in[0,1]$ and hence we can treat the values $v_{i}\left(y_{i}\right) \geq q_{i j} v_{i}\left(y_{i}^{\prime}\right)$ as described before the statement of the lemma.

- By the first set of constraints and definition of $y$, we have

$$
v_{i}\left(y_{i}\right) \geq \sum_{j \in D} q_{i j} u(i, j)+\sum_{j \in \mathcal{L} \backslash D} u(i, j) \geq \frac{1}{2} \sum_{j \in D} u(i, j)+\frac{1}{2} \sum_{j \in \mathcal{L} \backslash D} u(i, j) \geq \frac{1}{2} v_{i}\left(y^{\prime}\right) .
$$

Therefore, any feasible solution of the linear system in $q$ gives an allocation that satisfies the first condition of the lemma. Let us show that the system is indeed feasible. Namely, setting $q_{i j}=\frac{1}{2}$ for all $i \in \mathcal{A}^{\prime \prime}$ and all $j \in D$ we see that the above system is feasible. Since, the system is feasible we can also find a basic feasible solution $q$. By counting the number of tight constraints we show that there are at least $\left|\mathcal{L}^{+}\left(y^{\prime}\right)\right|-\left|\mathcal{L}_{1}\left(y^{\prime}\right)\right|-\left|\mathcal{A}^{\prime \prime}\right|$ zeros in $q$. Thus, allocation $y$ defined as $y_{i j}=q_{i j} y_{i j}^{\prime}$ will have support smaller by at least $\left|\mathcal{L}^{+}\left(y^{\prime}\right)\right|-\left|\mathcal{L}_{1}\left(y^{\prime}\right)\right|-\left|\mathcal{A}^{\prime \prime}\right|$.

The maximum number of (tight) constraints is obviously $\left|\mathcal{A}^{\prime \prime}\right|+|D|$. Therefore, $|\operatorname{supp}(q)| \leq$ $\left|\mathcal{A}^{\prime \prime}\right|+|D|$. Crucially, by the second constraint we have $\mathcal{L}^{+}(y)=\mathcal{L}^{+}\left(y^{\prime}\right)$. Hence, we only need to compare $\left|\operatorname{supp}\left(y^{\prime}\right)\right|$ and $|\operatorname{supp}(y)|$. The allocation $y^{\prime}$ has exactly $2|D|$ positive variables when restricted on $D$ and $\mathcal{A}^{\prime \prime}$. On the other hand, $q$ and therefore $y$ take at most $|D|+\left|\mathcal{A}^{\prime \prime}\right|$ nonzero values on $D$ and $\mathcal{A}^{\prime \prime}$. It follows that $y$ has at least $|D|-\left|\mathcal{A}^{\prime \prime}\right|$ less positive variables than $y^{\prime}$, i.e., $|\operatorname{supp}(y)| \leq\left|\operatorname{supp}\left(y^{\prime}\right)\right|-\left(|D|-\left|\mathcal{A}^{\prime \prime}\right|\right)$. By Corollary 5.8 and since $\left|\mathcal{A}^{\prime \prime}\right| \leq\left|\mathcal{A}^{\prime}\right|$ we have $|\operatorname{supp}(y)| \leq 2\left|\mathcal{A}^{\prime}\right|+2\left|\mathcal{L}^{+}\right|-\left|\mathcal{L}_{1}\left(y^{\prime}\right)\right|-|D|$. By recalling that $|D|=\left|\mathcal{L}^{+}\right|-\left|\mathcal{L}_{1}\left(y^{\prime}\right)\right|$ we get $|\operatorname{supp}(y)| \leq$ $2\left|\mathcal{A}^{\prime}\right|+\left|\mathcal{L}^{+}\right|$.

\section{Phase V: Rounding the mixed solution}

We present the rounding for a sparse solution of (Mixed+matching). We recall that by sparse we mean a feasible solution $(y, \pi)$ of (Mixed+matching) satisfying:

$$
\operatorname{supp}(y) \leq 2|\mathcal{A}|+\left|\mathcal{L}^{+}\right| \text {where } \mathcal{L}^{+}=\left\{j \in \mathcal{G} \backslash \mathcal{H}: \sum_{i \in \mathcal{A}^{\prime}} y_{i j}>0\right\}
$$


Such a sparse solution is rounded by setting $2|\mathcal{A}|$ positive variables in $y$ to 0 , i.e., a reduction of $(y, \pi)$ and allocating the items according to the support of the reduction. Formally, by a reduction of $(y, \pi)$ we mean a mixed integer solution $\left(y^{r}, \pi\right)$ obtained as follows (see Figure 1). For each item $j$ a fraction of which is allocated by $y$ (i.e., $j \in \mathcal{L}^{+}$), we pick an arbitrary agent $\kappa(j)$ getting the item (i.e., $y_{\kappa(j) j}>0$ ). We set $y_{\kappa(j) j}^{r}=y_{\kappa(j) j}$, and set $y_{i j}^{r}=0$ if $i \neq \kappa(j)$. In words, the agent $\kappa(j)$ keeps getting the same amount in reduction and no other agent receives any part of item $j$. By the bound on $\operatorname{supp}(y)$, this amounts to setting $\leq 2|\mathcal{A}|$ values $y_{i j}$ to 0 . Looking at the reduction from the agents perspective: let $d_{i}$ be the number of items agent $i$ lost by reduction, i.e., the number of items $j$ for which $y_{i j}>0$ and $y_{i j}^{r}=0$. Then, $\sum_{i \in \mathcal{A}^{\prime}} d_{i} \leq 2|\mathcal{A}|$.

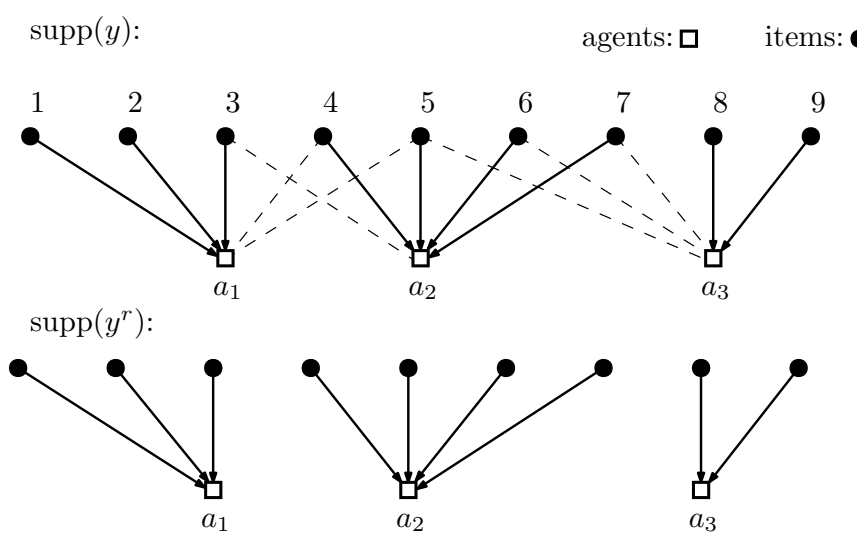

Figure 1: Support graph of an allocation $y$. Support graph of reduction $y^{r}$ obtained by $\kappa(1)=$ $\kappa(2)=\kappa(3)=a_{1}, \kappa(4)=\kappa(5)=\kappa(6)=\kappa(7)=a_{2}$, and $\kappa(8)=\kappa(9)=a_{3}$. It follows that $d_{a_{1}}=2$, $d_{a_{2}}=1$ and $d_{a_{3}}=3$.

The reduction $\left(y^{r}, \pi\right)$ might have an arbitrarily worse objective value than $(y, \pi)$ (e.g., if for agent $i$ we have $v_{i \pi(i)}=0$ and reduction sets $\left.y_{i}^{r}=0\right)$, but we show that we can find a different assignment $\rho$ such that $\left(y^{r}, \rho\right)$ is only worse by a constant factor than $(y, \pi)$, no matter how the reduction is carried out. The assignment $\rho$ is obtained as a combination of $\tau$ (the assignment obtained in Phase I) and $\pi$.

For a fixed reduction and the values $d_{i}, \rho$ and its properties are given by the following lemma.

Lemma 6.1 (Key rounding lemma). Let $\mathcal{H}$ be the set of most preferred items, $(y, \pi)$ a feasible solution to (Mixed+matching), and let $d_{i} \in \mathbb{N},\left(d_{i} \geq 1\right)$ for each $i \in \mathcal{A}$. In $O(|\mathcal{A}|)$ time, we can find an assignment $\rho$ such that

$$
\overline{\mathrm{NSW}}(y, \rho) \geq \frac{1}{2}\left(\prod_{i \in \mathcal{A}}\left(d_{i}+1\right)^{-w_{i}}\right)^{1 / \sum_{i \in \mathcal{A}} w_{i}} \overline{\mathrm{NSW}}(y, \pi)
$$

and for each $i \in \mathcal{A}$ it holds either

(a) $v_{i \rho(i)} \geq \frac{1}{d_{i}} v_{i}\left(y_{i}\right)$, or

(b) for each $j \in \mathcal{L}$ it holds $v_{i j} \leq \frac{1}{d_{i}+1}\left(v_{i}\left(y_{i}\right)+v_{i \rho(i)}\right)$.

Intuitively, the above lemma states that starting with a feasible allocation $y$, we can find an assignment $\rho$ that might have smaller $\overline{\operatorname{NSW}}(y, \rho)$ than $\overline{\operatorname{NSW}}(y, \pi)$ but has the following nice property for each agent $i \in \mathcal{A}$ : 
- In case (a), $i$ values the item $\rho(i)$ at least as she values a $1 / d_{i}$ fraction of $y_{i}$ (and thus at least a $1 /\left(d_{i}+1\right)$ fraction of $\left.v_{i}\left(y_{i}\right)+v_{i \rho(i)}\right)$. Hence, agent $i$ keeps a $1 /\left(d_{i}+1\right)$-fraction of her value just by keeping $\rho(i)$ even if we can take away all items $i$ gets from $\mathcal{L}$.

- In case (b), every item $\mathcal{L}$ has a small value for $i$ when compared to the combined value of $y_{i}$ and $\rho(i)$. That is, $i$ values $y_{i}$ and $\rho(i)$ significantly more than any $d_{i}$ items combined from $\mathcal{L}$. Looking at it from the other side, even if we were to take away any $d_{i}$ in $\mathcal{L}$ items from $i$ she will still keep a fraction of the value.

The essence of both cases is that the reduction will not hurt the agent too much. Before we present the proof of Lemma 6.1, we show that this is enough to prove Lemma 3.9.

Lemma 3.9. Let $\mathcal{H}$ be the set of most preferred items, and let $(y, \pi)$ be a solution to (Mixed + matching) as in Theorem 3.5. Let $\left(y^{r}, \pi\right)$ be a reduction of $(y, \pi)$. Then in polynomial-time we can find a matching $\rho: \mathcal{A} \rightarrow \mathcal{H}$ such that

$$
\overline{\mathrm{NSW}}\left(y^{r}, \rho\right) \geq \frac{1}{32 \gamma^{2}} \overline{\mathrm{NSW}}(y, \pi) .
$$

Further, if the valuations are linear, then we can find a matching $\rho: \mathcal{A} \rightarrow \mathcal{H}$ such that $\overline{\mathrm{NSW}}\left(y^{r}, \rho\right) \geq$ $\frac{1}{8} \overline{\mathrm{NSW}}(y, \pi)$.

Proof of Lemma 3.9. We first prove the lemma for the general case. Let $y^{r}$ be any reduction of $y$ and let $d_{i}$ be the number items agent $i$ lost in reduction. By sparsity in Theorem 3.5 we have $\sum_{i \in \mathcal{A}} d_{i} \leq 2|\mathcal{A}|$.

We use Lemma 6.1 to obtain $\rho$. Note that Lemma 6.1 requires $d_{i} \geq 1$ so we define $\bar{d}_{i}=$ $\max \left\{1, d_{i}\right\}$. Thus, now we have the bound $\sum_{i \in \mathcal{A}}\left(\bar{d}_{i}+1\right) \leq 4|\mathcal{A}|$. Let $\rho$ be the matching obtained by Lemma 6.1 given $\bar{d}_{i}$ 's and $y$. By Lemma 2.7 we have that

$$
\left(\prod_{i \in \mathcal{A}}\left(\bar{d}_{i}+1\right)^{-w_{i}}\right)^{1 / \sum_{i \in \mathcal{A}} w_{i}} \geq \frac{1}{4 \gamma} .
$$

Thus, $\overline{\mathrm{NSW}}(y, \rho) \geq \frac{1}{8 \gamma} \overline{\mathrm{NSW}}(y, \pi)$. By the same inequality, it suffices to show that $\overline{\mathrm{NSW}}\left(y^{r}, \rho\right) \geq$ $\left(\prod_{i \in A}\left(\bar{d}_{i}+1\right)^{-w_{i}}\right)^{\sum_{i \in \mathcal{A}} w_{i}} \overline{\mathrm{NSW}}(y, \rho)$. We do so, by showing that for each $i \in \mathcal{A}$ it holds $v_{i}\left(y_{i}^{r}\right)+$ $v_{i \rho(i)} \geq \frac{1}{\bar{d}_{i}+1}\left(v_{i}\left(y_{i}\right)+v_{i \rho(i)}\right)$. By Lemma 6.1 for agent $i$ we have either (a) or (b).

(a) In this case we have $\bar{d}_{i} v_{i \rho(i)} \geq v_{i}\left(y_{i}\right)$. Thus, $v_{i \rho(i)} \geq \frac{1}{\bar{d}_{i}+1}\left(v_{i}\left(y_{i}\right)+v_{i \rho(i)}\right)$. Consequently, $v_{i}\left(y_{i}^{r}\right)+v_{i \rho(i)} \geq \frac{1}{\bar{d}_{i}+1}\left(v_{i}\left(y_{i}\right)+v_{i \rho(i)}\right)$.

(b) We have $v_{i j} \leq \frac{1}{\bar{d}_{i}+1}\left(v_{i}\left(y_{i}\right)+v_{i \rho(i)}\right)$ for all $j \in \mathcal{L}$. Denote with $D_{i}$ the set of $d_{i}$ items $j$ for which $y_{i j}>0$ and $y_{i j}^{r}=0$. By subadditivity $v_{i}\left(D_{i}\right) \leq \sum_{j \in D_{i}} v_{i j}$. Therefore, $v_{i}\left(D_{i}\right) \leq$ $\frac{d_{i}}{\bar{d}_{i}+1}\left(v_{i}\left(y_{i}\right)+v_{i \rho(i)}\right) \leq \frac{\bar{d}_{i}}{\bar{d}_{i}+1}\left(v_{i}\left(y_{i}\right)+v_{i \rho(i)}\right)$. Hence, $v_{i}\left(y_{i}\right)-v_{i}\left(D_{i}\right)+v_{i \rho(i)} \geq \frac{1}{\bar{d}_{i}+1}\left(v_{i}\left(y_{i}\right)+v_{i \rho(i)}\right)$. By subadditivity and monotonicity we have $v_{i}\left(y_{i}^{r}\right) \geq v_{i}\left(y_{i}\right)-v_{i}\left(D_{i}\right)$, proving in this case as well that $v_{i}\left(y_{i}^{r}\right)+v_{i \rho(i)} \geq \frac{1}{\bar{d}_{i}+1}\left(v_{i}\left(y_{i}\right)+v_{i \rho(i)}\right)$. The lemma follows.

For additive valuations, we recall Theorem 3.6. It gives us an optimal solution of (EG) that is supported on a forest in which each tree contains an agent. In particular, this implies a nice property for the reductions of $y$. Namely, we can choose a reduction $y^{r}$ in which $d_{i} \leq 1$ for each agent $i \in \mathcal{A}$. Such a reduction is obtained by rooting each tree of the forest at an arbitrary agent and letting $\kappa(j)$ to be the parent agent of item $j$. Informally, each agent loses at most one item. Therefore, $\bar{d}_{i}=1$ for all $i \in \mathcal{A}$. The lemma follows by Lemma 6.1. 
The proof of Lemma 6.1 is presented in the following section.

\subsection{Constructing the new matching}

Recall Phase I where we defined $\tau$ as an assignment maximizing $\left(\prod_{i \in \mathcal{A}} v_{i \tau(i)}^{w_{i}}\right)$ and $\mathcal{H}$ the set of items assigned by $\tau$. We number the agents $\mathcal{A}=\{1,2, \ldots, n\}$, and renumber the items $\mathcal{H}=$ $\{1,2, \ldots, n\}$ such that $\tau=\{(i, i): i \in A\}$. In other words, $\tau$ assigns item $i \in \mathcal{G}$ to agent $i \in \mathcal{A}$.

Intuition We are given a feasible solution $(y, \pi)$ of (Mixed+matching) and $\tau$. For the sake of illustration assume that by using the matching $\tau$ instead of $\pi$ we don't lose too much in the objective, i.e.,

$$
\overline{\operatorname{NSW}}(y, \tau) \geq\left(\prod_{i \in \mathcal{A}}\left(d_{i}+1\right)^{-w_{i}}\right)^{1 / \sum_{i \in \mathcal{A}} w_{i}} \overline{\operatorname{NSW}}(y, \pi) .
$$

In this case, each agent $i$ gets the item $i$ from $\mathcal{H}$. Let us show that under the above assumption we can set $\rho=\tau$, i.e., that for each agent $i$ either (a) or (b) holds.

Claim 6.2. Let $i \in \mathcal{A}$. Then either $v_{i i}>\frac{1}{d_{i}} v_{i}\left(y_{i}\right)$ or for any $j \in \mathcal{L}$ it holds $v_{i j} \leq \frac{1}{d_{i}+1}\left(v_{i i}+v_{i}\left(y_{i}\right)\right)$

Proof of Claim. By the optimality of $\tau$ it then holds $v_{i i} \geq v_{i j}$ for all $j \in \mathcal{L}$. If $v_{i i} \geq \frac{1}{d_{i}} v_{i}\left(y_{i}\right)$ then (a) holds. Otherwise, we have that $d_{i} v_{i i}<v_{i}\left(y_{i}\right)$. Combining it with $v_{i j}<v_{i i}$, we have that

$$
\left(d_{i}+1\right) v_{i j} \leq\left(d_{i}+1\right) v_{i i}<v_{i}\left(y_{i}\right)+v_{i i}=v_{i}\left(y_{i}\right)+v_{i \tau(i)} .
$$

Therefore, our goal is to construct $\rho$ by "replacing" as much of $\pi$ with $\tau$ without losing too much in the objective. By Claim 6.2 for any agent for which $\rho(i)=\tau(i)$ we will have either (a) and (b). We formalize this idea below, and give a way of constructing $\rho$ such that even when $\rho(i) \neq \tau(i)$ still we have either (a) and (b).

Algorithm Let $(y, \pi)$ be a feasible solution of (Mixed+matching). We denote with $Y_{i}$ the value agent $i$ gets in $y$, i.e., $Y_{i}=v_{i}\left(y_{i}\right)$. We construct new assignment $\rho$ by combining $\pi$ and $\tau$. In particular, whenever $\pi(i)=\tau(i)$ then we set $\rho(i):=\pi(i)=\tau(i)$ and otherwise exactly one of the following will be the case: $\rho(i)=\tau(i), \rho(i)=\pi(i)$ or $\rho(i)=\emptyset$. Notation $\rho(i)=\emptyset$ represents the case that $i$ is not allocated any item from $\mathcal{H}$. (Formally, we can allocate one item to each agent since $|\mathcal{H}|=|\mathcal{A}|$ but as some agents might value some items at 0 it is simpler to say that agent is not allocated an item by $\rho$.)

Consider the symmetric difference of the two assignments $\pi \Delta \tau$. Each component is an alternating cycle; we consider the components one-by-one. Take any component $C$ of $\pi \Delta \tau$ with $c$ agents and $c$ items. Let the agents in the component be $a_{1}, a_{2}, \ldots, a_{c}$. The numbering is modulo $c: a_{c+k}=a_{k}$ for all $k \in \mathbb{Z}$. By the convention on the numbering, the corresponding items are also numbered $a_{1}, a_{2}, \ldots, a_{c}$, and $\left(a_{k}, a_{k}\right) \in \tau$ for all $k \in[c]$. We order the agents around the cycle such that $\left(a_{k}, a_{k-1}\right) \in \pi$ for all $k \in[c]$. Let $B:=B(C)=\left\{t \in[c]: Y_{a_{t}}>d_{a_{t}} v_{a_{t} a_{t-1}}\right\}$. We consider two cases based on the size of $B$ :

$|B|=0$. In this case we set $\rho\left(a_{t}\right)=\pi\left(a_{t}\right)=a_{t-1}$ for all $t \in[c]$.

$|B| \geq 1$. First, we trim $\pi$ by setting $\pi\left(a_{t}\right)=\emptyset$ for each $t \in B$. We have $\frac{Y_{a_{t}}+v_{a_{t} a_{t-1}}}{Y_{a_{t}}} \leq 2$ for each $t \in B$ since $d_{a_{t}} \geq 1$. In words, each agent losses at most half of her value.

After trimming $\pi$, the connected component $C$ decomposes into several alternating paths, see Figure 2. Consider one such path, starting in agent $a_{k}$ and ending in item $a_{r}$. It follows that 
$k \in B$ and $t \notin B$ for all $k<t \leq r$. We consider the following ratio that measures the change in the objective value by augmenting $\pi$ over the previously mentioned path:

$$
\varphi(C, k, r):=\left(\frac{Y_{a_{k}}}{v_{a_{k} a_{k}}+Y_{a_{k}}}\right)^{w_{a_{k}}} \prod_{t=k+1}^{r}\left(\frac{v_{a_{t} a_{t-1}}+Y_{a_{t}}}{v_{a_{t} a_{t}}+Y_{a_{t}}}\right)^{w_{a_{t}}} .
$$

If it holds that $\varphi(C, k, r) \leq \prod_{t=k}^{r-1}\left(d_{a_{t}}+1\right)^{w_{a_{t}}}$ then we say that the interval $[k, r]$ is reversible. Moreover, we set $\rho\left(a_{t}\right)=\tau\left(a_{t}\right)=a_{t}$ for all $k \leq t \leq r$. If $[k, r]$ is not reversible then we set $\rho\left(a_{k}\right)=\emptyset$ and $\rho\left(a_{t}\right)=\pi\left(a_{t}\right)=a_{t}$ for all $k<t \leq r$. We do the same for every augmenting path.

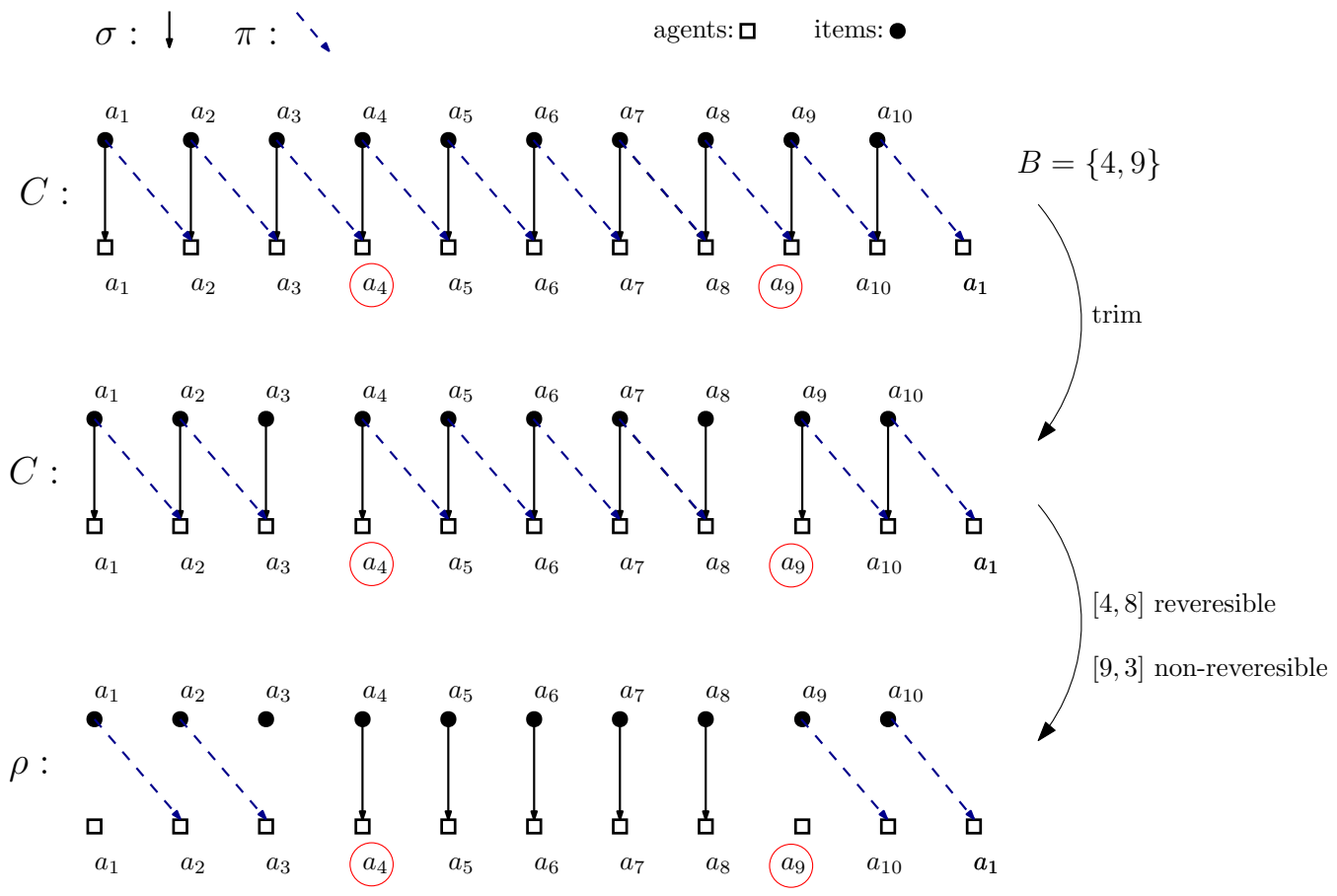

Figure 2: Assignments $\tau, \pi$, and $\rho$ resulting from $B=\{4,9\}$ and reversible interval $[4,8]$.

To prove Lemma 6.1, we first show that by changing the assignment from $\pi$ to $\rho$ the objective value of (Mixed+matching) cannot decrease by too much.

Lemma 6.3. The assignment $\rho$ can be constructed in linear time (in $n$ ), and it holds

$$
\frac{\overline{\mathrm{NSW}}(y, \pi)}{\overline{\mathrm{NSW}}(y, \rho)} \leq 2 \cdot\left(\prod_{i \in \mathcal{A}}\left(d_{i}+1\right)^{w_{i}}\right)^{1 / \sum_{i \in \mathcal{A}} w_{i}} .
$$

Proof. It suffices to prove the lemma for each of the connected components $C$ of $\pi \Delta \tau$. For $|B|=0$ the lemma holds trivially. So assume that $|B| \geq 1$ for the rest of the proof.

The procedure terminates in linear time, as we only require one pass through the agents and items in $C$. To prove the bound on $\frac{\overline{\mathrm{NSW}}(y, \rho)}{\overline{\mathrm{NSW}}(y, \pi)}$, we show that for every interval $[k, r]$ the objective value "before averaging" decreases at most by factor $2^{w_{a_{k}}} \prod_{t=k}^{r}\left(d_{a_{t}}+1\right)^{w_{a_{t}}}$. 
If interval $[k, r]$ is not reversible, then the change in the objective function is captured by $\left(\frac{v_{a_{k} a_{k-1}}+Y_{a_{k}}}{Y_{a_{k}}}\right)^{w_{a_{k}}}$, as for every agent $a_{t}$ with $t \in[k+1, r]$, we have $\rho\left(a_{t}\right)=\pi\left(a_{t}\right)$, and $\rho\left(a_{k}\right)=\emptyset$. Since $k \in B$, it follows that $Y_{a_{k}}>d_{a_{k}} v_{a_{k} a_{k-1}} \geq v_{a_{k} a_{k-1}}$. Thus, $\left(\frac{v_{a_{k} a_{k-1}}+Y_{a_{k}}}{Y_{a_{k}}}\right)^{w_{a_{k}}}<2^{w_{a_{k}}}$.

If, on the other hand, $[k, r]$ is reversible, then the difference in the objectives is captured by

$$
\left(\frac{v_{a_{k} a_{k-1}}+Y_{a_{k}}}{v_{a_{k} a_{k}}+Y_{a_{k}}}\right)^{w_{a_{k}}} \prod_{t=k+1}^{r}\left(\frac{v_{a_{t} a_{t-1}}+Y_{a_{t}}}{v_{a_{t} a_{t}}+Y_{a_{t}}}\right)^{w_{a_{t}}}=\left(\frac{v_{a_{k} a_{k-1}}+Y_{a_{k}}}{Y_{a_{k}}} \cdot \frac{Y_{a_{k}}}{v_{a_{k} a_{k}}+Y_{a_{k}}}\right)^{w_{a_{k}}} \prod_{t=k+1}^{r}\left(\frac{v_{a_{t} a_{t-1}}+Y_{a_{t}}}{v_{a_{t} a_{t}}+Y_{a_{t}}}\right)^{w_{a_{t}}}
$$

As $[k, r]$ is reversible $\varphi(C, k, r)=\left(\frac{Y_{a_{k}}}{v_{a_{k} a_{k}}+Y_{a_{k}}}\right)^{w_{a_{k}}} \cdot \prod_{t=k+1}^{r}\left(\frac{v_{a_{t} a_{t-1}}+Y_{a_{t}}}{v_{a_{t} a_{t}}+Y_{a_{t}}}\right)^{w_{a_{t}}}<\prod_{t=k}^{r}\left(d_{a_{t}}+1\right)^{w_{a_{t}}}$. Since $k \in B$ and $d_{a_{k}} \geq 1$ we again have $\frac{v_{a_{k} a_{k-1}}+Y_{a_{k}}}{Y_{a_{k}}}<2$. Hence, the change in the objective value is bounded by $2^{w_{a_{k}}} \cdot \prod_{t=k}^{r}\left(d_{a_{t}}+1\right)^{w_{a_{t}}}$.

It is left to show that for each agent $i$ either (a) or (b) holds. Recall that $Y_{i}=v_{i}\left(y_{i}\right)$.

Lemma 6.4. Let $i \in \mathcal{A}$. Then we either have

(a) $v_{i \rho(i)} \geq \frac{1}{d_{i}} v_{i}\left(y_{i}\right)$, or

(b) for each $j \in \mathcal{L}$ it holds $v_{i j} \leq \frac{1}{d_{i}+1}\left(v_{i}\left(y_{i}\right)+v_{i \rho(i)}\right)$.

To prove the lemma we use the following simple claim, which can applied to any agent $i \notin B$ :

Claim 6.5. For any agent $i \in \mathcal{A}$, if $Y_{i} \leq d_{i} v_{i \pi(i)}$, then $\frac{v_{i \pi(i)}+Y_{i}}{v_{i i}+Y_{i}} \leq \frac{\left(d_{i}+1\right) v_{i \pi(i)}}{v_{i i}}$.

Proof of Lemma 6.4. If $\rho(i)=i$, that is, agent $i$ receives the same item in $\rho$ as in $\tau$ then the lemma follows by Claim 6.2. For the rest of the proof we assume $\rho(i) \neq i$. Hence, either $\rho(i)=\pi(i)$ or $\rho(i)=\emptyset$.

We consider the component $C$ of $\tau \Delta \pi$ containing an agent $i$. We use the notation as before, denoting the agents in $C$ by $a_{1}, a_{2}, \ldots, a_{c}$, and letting $i=a_{k}$.

If $\rho\left(a_{k}\right)=\pi\left(a_{k}\right)=a_{k-1}$ then for $i$ it holds (a). Namely, $\rho\left(a_{k}\right)=a_{k-1}$ implies that $k \notin B$ as otherwise this would be trimmed. Thus $Y_{a_{k}} \leq d_{a_{k}} v_{a_{k} a_{k-1}}$; or equivalently $v_{a_{k} a_{k-1}} \geq \frac{1}{d_{a_{k}}} Y_{a_{k}}$.

If on the other hand $\rho\left(a_{k}\right)=\emptyset$, we have that $k \in B$ and also that the interval $[k, r]$ with starting and $k$ and ending in $r$ that corresponds to some alternating path in $C$ is not reversible (otherwise, $\left.\rho\left(a_{k}\right)=a_{k}\right)$. Therefore, $\varphi(C, k, r)>\prod_{t=1}^{r}\left(d_{a_{t}}+1\right)^{w_{a_{t}}}$. Recall that for each such considered interval we have $k \in B$ and $t \notin B$. Starting with $\prod_{t=k}^{r}\left(d_{a_{t}}+1\right)^{w_{a_{t}}}<\varphi(C, k, r)$ and then by Claim 6.5 we obtain

$$
\begin{aligned}
1 & <\prod_{t=k}^{r-1}\left(d_{a_{t}}+1\right)^{-w_{a_{t}}} \cdot\left(\frac{Y_{a_{k}}}{v_{a_{k} a_{k}}+Y_{a_{k}}}\right)^{w_{a_{k}}} \cdot \prod_{t=2}^{r}\left(\frac{v_{a_{t} a_{t-1}}+Y_{a_{t}}}{v_{a_{t} a_{t}}+Y_{a_{t}}}\right)^{w_{a_{t}}} \\
& \leq\left(d_{a_{k}}+1\right)^{-w_{a_{k}}} \cdot\left(\frac{Y_{a_{k}}}{v_{a_{k} a_{k}}+Y_{a_{k}}}\right)^{w_{a_{k}}} \cdot \prod_{t=2}^{r}\left(\frac{v_{a_{t} a_{t-1}}}{v_{a_{t} a_{t}}}\right)^{w_{a_{t}}} .
\end{aligned}
$$

We further bound

$$
1<\left(d_{a_{k}}+1\right)^{-w_{a_{k}}} \cdot\left(\frac{Y_{a_{k}}}{v_{a_{k} j}} \cdot \frac{v_{a_{k} j}}{v_{a_{k} a_{k}}}\right)^{w_{a_{k}}} \cdot \prod_{t=2}^{r}\left(\frac{v_{a_{t} a_{t-1}}}{v_{a_{t} a_{t}}}\right)^{w_{a_{t}}} .
$$


By the optimal choice of $\tau$, for every $j \in \mathcal{L}$ we have

$$
1 \leq\left(\frac{v_{a_{k} a_{k}}}{v_{a_{k} j}}\right)^{w_{a_{k}}} \cdot \prod_{t=2}^{r}\left(\frac{v_{a_{t} a_{t}}}{v_{a_{t} a_{t-1}}}\right)^{w_{a_{t}}}
$$

Combining the last two inequalities, we obtain $Y_{a_{k}}>\left(d_{a_{k}}+1\right) v_{a_{k} j}$. Hence, in this case (b) holds, by recalling that $i=a_{k}$ and $\rho\left(a_{k}\right)=\emptyset$.

\section{Rado valuations and $M^{\natural}$-concave functions}

In this section, we prove Lemma 2.5 showing that Rado valuations are $\mathrm{M}^{\natural}$-concave and Lemma 7.2 showing that the function $\nu$ as defined in Theorem 2.6 is indeed the continuous closure of the Rado valuation. We also discuss related conjectures on constructive characterizations of $\mathrm{M}^{\natural}$-concave functions.

\subsection{Rado valuations are $\mathrm{M}^{\natural}$-concave}

Rado valuations turn out to be a special case of a more general construction described in [50], called 'transformation by networks'. We now present it in the special case when the network is a bipartite graph (instead of a directed graph) with linear edge costs (instead of concave functions), and the functions are restricted to the binary domain (instead of the nonnegative integer lattice).

Theorem 7.1 ([50, Chapter 9.6.1],[53, Section 6.2]). Let $H=\left(\mathcal{G}, V^{\prime} ; E^{\prime}\right)$ be a bipartite graph with cost function $c^{\prime}: E^{\prime} \rightarrow \mathbb{R}_{+}$. Given an $M^{\natural}$-concave function $g:\{0,1\}^{V^{\prime}} \rightarrow \mathbb{R} \cup\{-\infty\}$ the following function $\tilde{g}:\{0,1\}^{\mathcal{G}} \rightarrow \mathbb{R}$ is also $M^{\natural}$-concave:

$$
\tilde{g}(x)=\max _{y \in\{0,1\}^{V^{\prime}}, z \in\{0,1\}^{\mathcal{G}} \times V^{\prime}}\left\{g(y)+c^{\prime}(z): \sum_{k \in V^{\prime}} z_{k j}=x_{j}, \forall j \in \mathcal{G} \text { and } \sum_{j \in \mathcal{G}} z_{k j}=y_{k}, \forall k \in V^{\prime}\right\} .
$$

We use the transformation to show that the Rado valuation functions are $\mathrm{M}^{\natural}$-concave.

Lemma 2.5. Every Rado valuation $v: 2^{\mathcal{G}} \rightarrow \mathbb{R}_{+}$is an $M^{\natural}$-concave function.

Proof. Consider a Rado valuation $v$ as in Definition 2.4 , given by a bipartite graph $(\mathcal{G}, V ; E)$ with a cost function $c: E \rightarrow \mathbb{R}$ on the edges, and a matroid $\mathcal{M}=(V, \mathcal{I})$ with rank function $r$.

Let us define $V^{\prime}=V \cup D$ with a set $D$ of $|\mathcal{G}|$ dummy nodes. Let $E^{\prime}$ be the union of $E$ and a perfect matching of dummy edges between $\mathcal{G}$ and $D$. Let $c_{e}^{\prime}=c_{e}$ for all $e \in E$ and $c_{e}^{\prime}=0$ on all new dummy edges. Let us define a matroid $\mathcal{M}^{\prime}=\left(V^{\prime}, \mathcal{I}^{\prime}\right)$ where a set $S \in \mathcal{I}^{\prime}$ if and only if $S \cap V \in \mathcal{I}$; that is, we add the dummy elements in $D$ freely to the matroid. We define

$$
g(y)= \begin{cases}0 & \text { if } y=\chi_{S} \text { for some } S \in \mathcal{I}^{\prime} \\ -\infty & \text { otherwise }\end{cases}
$$

As the indicator function of the independent sets of a matroid, $g$ is well-known to be $\mathrm{M}^{\natural}$-concave (see e.g. [50, Section 4.7]). We claim that $\tilde{g}$ defined in (6) equals the Rado valuation $v$.

First, for $S \subseteq \mathcal{G}$, let $M$ be the maximum cost matching in the definition of $v(S)$. We can extend this to a perfect matching $M^{\prime}$ with $\delta_{S}\left(M^{\prime}\right)=S$ by adding dummy edges incident to the nodes in $S \backslash \delta_{S}(M)$. We then define $z=\chi_{M^{\prime}}$ and $y=\chi_{\delta_{V^{\prime}}\left(M^{\prime}\right)}$. Thus, $\tilde{g}\left(\chi_{S}\right) \geq g(y)+c^{\prime}(z)=0+c(M)=v(S)$. Conversely, consider the optimal $(y, z)$ in the definition of $\tilde{g}\left(\chi_{S}\right)$. By the above bound, we know that $\tilde{g}\left(\chi_{S}\right) \geq v(S) \geq 0$ is finite, and therefore $g(y)=0$. The set of (non-dummy) edges $e \in E$ with $z_{e}=1$ thus form a matching with $\delta_{V}(M) \in \mathcal{I}, \delta_{\mathcal{G}}(M) \subseteq S$, and $c(M)=\tilde{g}\left(\chi_{S}\right)$, showing that $\tilde{g}\left(\chi_{S}\right) \leq v(S)$. 


\subsection{Concave closure of Rado valuations}

We now complete the proof of Theorem 2.6, showing that the function $\nu$ defined in (2) is indeed the continuous extension of the Rado valuation $v$.

The value $\bar{g}(x)$ for $x \in[0,1]^{m}$ is defined by a linear program (1). ${ }^{8}$ In the proof, we will use the dual LP:

$$
\begin{aligned}
& \begin{aligned}
\bar{v}(x)=\min & p^{\top} x+\alpha \\
\text { s.t.: } & p(S)+\alpha \geq v(S) \quad \forall S \subseteq \mathcal{G}
\end{aligned} \quad \max \sum_{S \subseteq G} \lambda_{S} v(S) \\
& (p, \alpha) \in \mathbb{R}^{m+1} \quad \text { s.t.: } \quad \sum_{S \subseteq G} \lambda_{S} \chi_{S}=x \\
& \sum_{S \subseteq G} \lambda_{S}=1 \\
& \lambda \geq 0
\end{aligned}
$$

Lemma 7.2. Let $v$ be a Rado valuation given by a bipartite graph $(\mathcal{G}, V ; E)$ with costs on the edges $c: E \rightarrow \mathbb{R}$, and a matroid $\mathcal{M}=(V, \mathcal{I})$ with a rank function $r=r_{\mathcal{M}}$ as in Definition 2.4. Let $\nu(x)$ be the function defined in (2), that is,

$$
\begin{array}{cc}
\nu(x):=\max \sum_{(j, k) \in E} c_{j k} z_{j k} & \\
\text { s.t.: } \quad \sum_{k \in V} z_{j k} \leq x_{j} \quad \forall j \in \mathcal{G} \\
\sum_{j \in \mathcal{G}, k \in T} z_{j k} \leq r(T) \quad \forall T \subseteq V \\
z \geq 0 .
\end{array}
$$

Then, $\bar{v}(x)=\nu(x)$ holds for every $x \in \mathbb{R}_{+}^{m}$.

Proof. We let $\mathcal{M}(x)$ denote the set of feasible solutions of (2). Fix any $x \in \mathbb{R}^{m}$. We first show that $\bar{v}(x) \leq \nu(x)$.

Consider an optimal solution $\lambda$ for the dual LP in (8) such that $\bar{v}(x)=\sum_{S \subseteq \mathcal{G}} \lambda_{S} v(S)$. For every $S \subseteq \mathcal{G}$, we have an integral allocation $M_{S}$ of the goods in $\mathcal{M}\left(\chi_{S}\right)$ that is optimal in the linear program (2) defining $\nu\left(\chi_{S}\right)=v(S)$; these two are equal using Theorem 2.6. It is easy to see that $\sum_{S \subset \mathcal{G}} \lambda_{S} M_{S} \in \mathcal{M}(x)$. Thus, $\bar{v}(x) \leq \nu(x)$.

For the other direction $\bar{v}(x) \geq \nu(x)$, let $z$ be the optimal solution defining $\nu(x)$ in (2). By the integrality of the bipartite matching polytope, we can write the fractional matching $z$ as a convex combinations of integral allocations $M_{S}$ for $S \subseteq \mathcal{G}$, i.e., $z=\sum_{S \subseteq \mathcal{G}} \lambda_{S} M_{S}$ for some $\lambda \geq 0$ with $\sum \lambda_{S}=1$. The dual of (2) is

$$
\begin{array}{ll}
\min & \sum_{j \in \mathcal{G}} x_{j} \pi_{j}+\sum_{T \subseteq V} \rho_{T} \\
\text { s.t.: } & \pi_{j}+\sum_{T: k \in T} \rho_{T} \geq c_{j k} \quad \forall j \in \mathcal{G}, \forall T \subseteq V \\
& \pi \in \mathbb{R}_{+}^{\mathcal{G}}, \quad \rho \in \mathbb{R}_{+}^{2^{V}} .
\end{array}
$$

\footnotetext{
${ }^{8}$ For $\mathrm{M}^{\natural}$-concave functions defined over the lattice $\mathbb{Z}^{n}$, the definition of the extension includes a constraint for every lattice point, thus, the system is not finite. Still, it can be described by a 'local' linear program, see [50, (3.64) and Theorem 6.42].
} 
Consider an optimal dual solution $(\pi, \rho)$. By complementarity, $\pi_{i}+\sum_{S: k \in T} \rho_{T}=c_{j k}$ for every $(j, k) \in \operatorname{supp}(z)$; if $\rho_{T}>0$ for $T \subseteq V$ then $z(\delta(T))=r(T)$, and if $\pi_{j}>0$ for $j \in \mathcal{G}$ then $z(\delta(j))=x_{j}$.

Since $z=\sum_{S} \lambda_{S} M_{S}$, we have $M_{S} \subseteq \operatorname{supp}(z)$, and $\delta_{M_{S}}(S)=r(S)$ whenever $z(\delta(S))=r(S)$. Further, $z(\delta(j))=x_{j}$ implies that every matching $M_{S}$ with $j \in S$ covers $j$. We see that $\chi_{M_{S}}$ and $(\pi, \rho)$ satisfy complementary slackness in (2) for every set $S$ with $\lambda_{S}>0$. Thus, $c\left(M_{S}\right)=\nu\left(\chi_{S}\right)$, and $\nu\left(\chi_{S}\right)=v(S)$ again by Theorem 2.6. We can thus conclude that

$$
\nu(x)=\sum_{S \subseteq \mathcal{G}} \lambda_{S} c\left(M_{S}\right)=\sum_{S \subseteq \mathcal{G}} \lambda_{S} v(S) \leq \bar{v}(x),
$$

completing the proof.

\subsection{Conjectures on characterizing $M^{\natural}$-concave functions}

First, we answer Frank's question negatively, showing that Rado valuations do not cover the entire class of $\mathrm{M}^{\natural}$-concave valuations. Lehmann, Lehmann, and Nisan [45, Example 1] gave an example that is an $\mathrm{M}^{\natural}$-concave (gross substitutes) valuation but not OXS. We show that the same example is also not a Rado valuation; the proof is similar.

Lemma 7.3. Consider the following valuation on the ground set $\mathcal{G}=\{1,2,3,4\}$. We define $v(S)=10$ if $|S|=1$, and $v(S)=19$ for all sets with $|S| \geq 2$ except $v(\{1,3\})=v(\{2,4\})=15$. This is $M^{\natural}$-concave, but not a Rado valuation function.

Proof. The proof that $v$ is a gross substitutes $/ \mathrm{M}^{\natural}$-concave valuation is given in [45, Claim 2]. Let us show that it is not a Rado valuation. For a contradiction, assume $v$ is a Rado valuation as in Definition 2.4. We can assume that the matroid on $V$ does not contain any loops (rank-0 elements), and any parallel elements, i.e., any set $S \subseteq V$ with $|S| \geq 2$ and $r(S)=1$; we can contract any such set to a single element and obtain another representation.

Trivially, we can assume that no edge in the bipartite graph $(\mathcal{G}, V ; E)$ has cost more than 10. By $v(\{1\})=10$ we have an element $u \in V$ with $c_{1 u}=10$. Since $v(\{2\})=10$, there is $u^{\prime} \in V$ such that $c_{2 u^{\prime}}=10$. Since $v(\{1,2\})<20$ we have $u^{\prime}=u$ as otherwise $(1, u),\left(2, u^{\prime}\right)$ would be an independent matching of cost 20, since $r\left(\left\{u, u^{\prime}\right\}\right)=2$ by the above assumption.

An analogous argument shows that $c_{j u}=10$ for all $j \in\{1,2,3,4\}$. We must have $c_{j k} \leq 5$ for any $j \in\{1,2,3,4\}$ and any $k \in V \backslash\{v\}$, as otherwise we would have an independent matching of cost $>15$ covering $\{1,3\}$ or $\{2,4\}$, again using the assumption of no parallel elements in $V$. Now, it is clear that we cannot realize $v(\{1,2\})=19$.

The reason why Rado valuations are not a rich enough class is that it is not closed under endowment operations. Given a valuation $v: 2^{\mathcal{G}} \rightarrow \mathbb{R}$ and a subset $T \subseteq \mathcal{G}$, we can define the valuation $v^{\prime}: 2^{\mathcal{G}^{\prime} \backslash T} \rightarrow \mathbb{R}_{+}$as

$$
v^{\prime}(X)=v(X \cup T)-v(T) .
$$

Using Definition 2.2, it is immediate that if $v$ is $\mathrm{M}^{\natural}$-concave than so is $v^{\prime}$. It is not difficult to check that the example in Lemma 7.3 arises as the endowment of a Rado valuation, showing that Rado valuations are not closed under endowment operations.

Endowment can be seen as a minor operation. Let us say that $v$ is a Rado minor valuation if it arises from a Rado valuation by the endowment operation. Note that this class is trivially closed for endowment. This motivates the following conjecture:

Conjecture 7.4. Every $M^{\natural}$-concave valuation arises as a Rado minor valuation. 
Ostrovsky and Paes Leme [59] previously posed the following stronger "matroid based valuation conjecture". We define the merging/convolution of the valuations $v_{1}, v_{2}: 2^{\mathcal{G}} \rightarrow \mathbb{R}$ as

$$
v^{*}(S)=\max _{T \subseteq S} v_{1}(T)+v_{2}(S \backslash T) \quad \forall S \subseteq \mathcal{G} .
$$

Merging two $\mathrm{M}^{\natural}$-concave functions results in an $\mathrm{M}^{\natural}$-concave function.

Conjecture 7.5 (Ostrovsky and Paes Leme [59]). Every $M^{\natural}$-concave valuation arises by the repeated application of endowment and merging operations starting from weighted matroid rank functions.

This conjecture is still open. Tran [67] showed that only allowing merging above is not sufficient, even if starting from a slightly broader class also including partition valuations.

Conjecture 7.4 is a natural weakening of Conjecture 7.5: weighted matroid rank functions form a subclass of Rado valuations, and it is easy to verify that Rado minor valuations are closed under merging and endowment. However, Conjecture 7.4 may be more plausible, since the class of Rado valuations appears to be much richer than that of weighted rank functions.

Balkanski and Paes Leme [5] gave a negative answer to the question whether every $\mathrm{M}^{\natural}$-concave valuation arises as a conic combination of (unweighted) matroid rank functions. Note that $\mathrm{M}^{\natural}$ concave functions are not closed under conic combinations, even the sum of two matroid rank functions may not be $\mathrm{M}^{\natural}$-concave. Thus, the questions was whether conic combinations of matroid ranks forms a superclass of the $\mathrm{M}^{\natural}$-concave valuations.

\section{Connection to spending restricted equilibrium}

The first constant factor approximation algorithm for the Nash social welfare problem was given by Cole and Gkatzelis [17] using the so-called spending restricted (SR) equilibrium. Since then, the SR-equilibrium is one of the main concepts used in the design of the approximation algorithms for the NSW problem $[2,18,28,29]$.

An important feature of the SR-equilibrium is that the items highly valued by the agents are recognized as items with price more than 1 (expensive) in the equilibrium. Isolating such items is at the essence of the approximation algorithms in the literature. The main idea is that each of the expensive items must be allocated integrally to one agent only, thereby preventing the unbounded integrality gap arising when several agents share a very desirable good, see [17, Lemma 3.1].

In this section, we illustrate a connection between the approach we use and the SR-equilibrium. In that light, for the rest of the section we focus on the case of symmetric Nash social welfare problem where agents have additive valuations. We show that the set of the most preferred items $\mathcal{H}$ obtained in Phase I contains all the expensive items in an SR-equilibrium. Similarly to the algorithms relying on the SR-equilibrium where expensive items have special status during rounding, the items in $\mathcal{H}$ are allocated integrally throughout our algorithm. Intuitively, this is how we are overcoming the unbounded integrality gap.

SR-equilibrium We quickly recall the necessary definitions and refer the reader to [17] for more details. The market consists of a set of divisible items $\mathcal{G}$, agents $\mathcal{A}$ each of which has a budget of 1 and an additive valuation function over the items. A valuation of agent $i$ is additive if her value is given as $v_{i}\left(x_{i}\right)=\sum_{j \in \mathcal{G}} v_{i j} x_{i j}$ for all $x_{i} \in \mathbb{R}_{+}^{\mathcal{G}}$ and $v_{i j} \in \mathbb{R}_{+}$.

Consider prices $p \in \mathbb{R}_{+}^{\mathcal{G}}$ for the items in $\mathcal{G}$. We say that an item $j$ is maximum bang per buck (MBB) for agent $i$ if $j \in \arg \max _{j \in \mathcal{G}}\left\{v_{i j} / p_{j}\right\}$. For an allocation $x$, the spending of an agent on $x_{i}$ is $p^{\top} x_{i}$ and the spending on an item $j$ is $\sum_{i \in \mathcal{A}} p_{j} x_{i j}$. The MBB items are exactly the items an agent would buy at prices $p$ in order to maximize its valuation such that spending is not more than a given budget. 
Definition 8.1. A spending restricted (SR) equilibrium is a fractional allocation $x$ and a price vector $p$ such that every agent spends all of her budget on her MBB items at prices $p$, and the total spending on each item is equal to $\min \left\{1, p_{j}\right\}$.

By scaling the valuation of each agent we can assume that the maximum bang per buck is one for all agents. Under such a scaling, in an SR-equilibrium we also have that $v_{i j}=p_{j}$ whenever item $j$ is MBB for agent $i$ and $v_{i j}<p_{j}$ otherwise. We work with this assumption for the rest of this section.

NSW and SR-equilibrium Consider a NSW welfare instance with items $\mathcal{G}$ and agents $\mathcal{A}$ where each agent $i$ has additive valuation. For the NSW problem, the valuations are discrete function and the value of a subset of items $S$ for agent $i$ is given by $v_{i}(S)=\sum_{j \in S} v_{i j}$. The extension of an additive valuation $v_{i}$ to $\mathbb{R}_{+}^{\mathcal{G}}$ is naturally defined as $v_{i}\left(x_{i}\right)=\sum_{j \in \mathcal{G}} v_{i j} x_{i j}$ for all $x_{i} \in \mathbb{R}_{+}^{\mathcal{G}}$. We construct the market from the NSW instance from the same set of items $\mathcal{G}$ that are now declared divisible and the set of agents $\mathcal{A}$ each equipped with the extension of the discrete additive valuation and budget one.

Let $(x, p)$ be an SR-equilibrium in such a market. Define the set of expensive goods $\bar{H}$ as $\bar{H}:=\left\{j \in \mathcal{G}: p_{j}>1\right\}$. Cole and Gkatzelis [17] proved that $\left(\prod_{j \in \bar{H}} p_{j}\right)^{1 /|\mathcal{A}|}$ is an upper-bound on the optimal value of NSW, and gave a rounding algorithm that uses an SR-equilibrium as a starting point.

In the next lemma we show that $\bar{H} \subseteq \mathcal{H}$, where $\mathcal{H}$ is the set of the most preferred goods obtained in Phase $\mathbf{I}$ of our algorithm. Recall that $\tau$ is an assignment maximizing $\left(\prod_{i \in \mathcal{A}} v_{i \tau(i)}\right)^{1 /|\mathcal{A}|}$ and that $\mathcal{H}:=\tau(A)$. In words, $\tau$ maximizes the NSW welfare under the constraint that each agent gets exactly one item.

For the purposes of the proof recall that the the spending graph $\left(\mathcal{A}, \mathcal{G} ; E_{x}\right)$ of an allocation $x$ is defined as $i j \in E_{x}$ if and only if $x_{i j}>0$.

Lemma 8.2. It holds $\bar{H} \subseteq \mathcal{H}$.

Proof. Using a cycle canceling argument, we can assume that the spending graph of SR-equilibrium $(x, p)$ is a forest $F$. Moreover, since $x$ is an SR-equilibrium allocation, every tree contains at least one agent and one item. The next claim states that an expensive item is a leaf in some tree in $F$ only in a very special case.

Claim 8.3. Let $T=\left(\mathcal{A}_{1}, \mathcal{G}_{1} ; E_{1}\right)$ be a tree component of $F$ and $j \in \mathcal{G}_{1}$ an item in $T$. If $p_{j}>1$ then either $\left|A_{1}\right|=\left|\mathcal{G}_{1}\right|=1$ or $j$ is not a leaf of $T$.

Proof of Claim. By definition of SR-equilibrium each agent spends all of her budget which is 1 . If $j$ is a leaf, then there is unique agent $i$ buying $j$. Moreover, $i$ spends all 1 unit of her budget on $j$ and cannot buy any other item. Thus, $\mathcal{A}_{1}=\{i\}$ and $\mathcal{G}_{1}=\{j\}$.

Let $\kappa: \bar{H} \rightarrow \mathcal{A}$ such that $x_{\kappa(j) j}>0$. Such an function $\kappa$ exists by definition of SR-equilibrium. Moreover, by Claim 8.3 we can choose $\kappa$ to be an assignment (root every tree of $F$ in an arbitrary item and assign the expensive item to any child agent). We are ready to prove the lemma.

For the sake of contradiction suppose that there is an item $j_{1} \in \bar{H}$ such that $j_{1} \notin \mathcal{H}$. In other words, $p_{j_{1}}>1$ and $j_{1}$ is not allocated to any agent by $\tau$. By definition we have $\bar{H} \leq|\mathcal{A}|=|\mathcal{H}|$. Consider the component of the symmetric difference $\tau \Delta \kappa$ containing $j$. Since $j_{1} \notin \mathcal{H}$ and $\mathcal{H}=\tau(\mathcal{A})$, this component forms a path starting in $j_{1}$ and ending in a vertex $j_{k+1}$ in $\mathcal{G} \backslash \bar{H}$; see Figure 3 . Let us denote the path as $j_{1}, \kappa\left(j_{1}\right), j_{2}, \kappa\left(j_{2}\right), \ldots, \kappa\left(j_{k}\right), j_{k+1}$ where $j_{t+1}=\tau\left(\kappa\left(j_{t}\right)\right)$ for $t \in[k]$, and $j_{t} \in \bar{H}$ for $t \leq k$. 


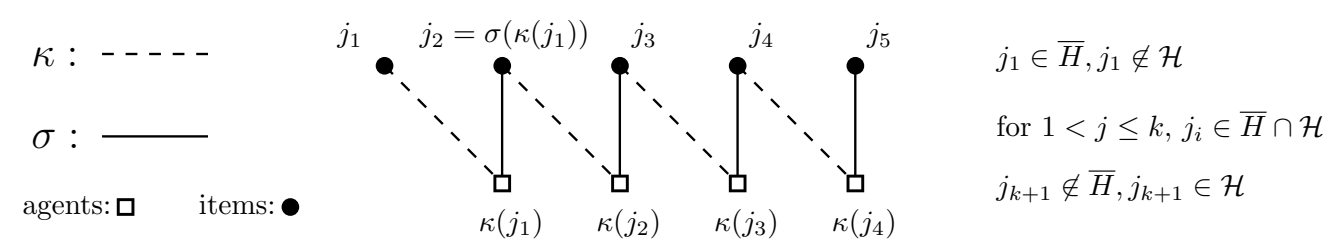

Figure 3: A component of $\kappa \Delta \tau$ containing $j_{1}$.

Recall, that MBB of each agent is one, therefore $v_{i j}=p_{j}$ for each $i, j$ with $x_{i j}>0$. By definition of $\kappa$ we have that $v_{\kappa\left(j_{t}\right) j_{t}} \geq v_{\kappa\left(j_{t}\right) j_{t+1}}$ for $t \in[k-1]$. Moreover, we have $v_{\kappa\left(j_{1}\right) j_{1}}=p_{j_{1}}>1 \geq p_{j_{k+1}} \geq$ $v_{\kappa\left(j_{k}\right), k+1}$. Since $j_{t+1}=\tau\left(\kappa\left(j_{t}\right)\right)$, augmenting over the above path will contradict the optimality of $\tau$.

\section{Conclusions and future work}

We have given a constant factor approximation algorithm for the Nash social welfare problem with Rado valuations, assuming that the weights of the agents are bounded by a constant. Rado valuations form a broad subclass of gross substitutes valuations. It remains open to obtain a constant factor approximation for the entire class of gross substitutes valuations, and for even more general classes, such as submodular valuations. The other main open question is to remove the assumption of bounded weights, that is, to obtain a constant factor independent of the parameter $\gamma$.

We note that for subadditive valuations, Barman, Bhaskar, Krishna, and Sundaram [9] gave an $O(n)$-approximation and showed that this is essentially tight: an $O\left(n^{1-\varepsilon}\right)$ approximation would require an exponential number of oracle queries for any fixed $\varepsilon>0$.

The algorithm is based on a mixed integer programming relaxation, and decomposes into a number of phases. Most reduction steps are applicable for the general subadditive setting. We only require Rado valuations for Phase IV, to obtain an approximate solution with a small support. The factor $\gamma$ only appears in the reduction in Phase II, where we restrict each agent to receiving only a single item from the set $\mathcal{H}$. Besides extending the result to more general settings, there is much scope for improving the approximation factor by using tighter analyses and amortizing across the different phases.

For example, we expect that a (mild) extension to budget-Rado valuations should be achievable. Similarly to $[15,28]$, this means Rado valuations with a cap on the maximum obtainable value for each agent. This only requires a slightly more careful argument in Phase IV.

Our work also highlights Rado valuations as an interesting class of gross substitutes valuations; this could be relevant also for other problems in mechanism design: it is a broad class including most common examples such as weighted matroid rank functions and OXS valuations, yet it has a rich combinatorial structure that can be exploited for algorithm design.

\section{References}

[1] Nima Anari, Shayan Oveis Gharan, Amin Saberi, and Mohit Singh. Nash social welfare, matrix permanent, and stable polynomials. In Proceedings of the 8th Innovations in Theoretical Computer Science Conference (ITCS), volume 67, page 36. Schloss Dagstuhl-Leibniz-Zentrum fuer Informatik, 2017.

[2] Nima Anari, Tung Mai, Shayan Oveis Gharan, and Vijay V Vazirani. Nash social welfare for indivisible items under separable, piecewise-linear concave utilities. In Proceedings of the 29th 
annual ACM-SIAM Symposium on Discrete Algorithms (SODA), pages 2274-2290. SIAM, 2018.

[3] Chidambaram Annamalai, Christos Kalaitzis, and Ola Svensson. Combinatorial algorithm for restricted max-min fair allocation. ACM Transactions on Algorithms (TALG), 13(3):1-28, 2017.

[4] Arash Asadpour and Amin Saberi. An approximation algorithm for max-min fair allocation of indivisible goods. SIAM Journal on Computing (SICOMP), 39(7):2970-2989, 2010.

[5] Eric Balkanski and Renato Paes Leme. On the construction of substitutes. Mathematics of Operations Research, 45(1):272-291, 2020.

[6] Nikhil Bansal and Maxim Sviridenko. The Santa Claus problem. In Proceedings of the 38th ACM Symposium on Theory of Computing (STOC), pages 31-40, 2006.

[7] Julius B Barbanel. The geometry of efficient fair division. Cambridge University Press, 2005.

[8] Siddharth Barman, Sanath Kumar Krishnamurthy, and Rohit Vaish. Finding fair and efficient allocations. In Proceedings of the 2018 ACM Conference on Economics and Computation (EC), pages 557-574. ACM, 2018.

[9] Siddharth Barman, Umang Bhaskar, Anand Krishna, and Ranjani G Sundaram. Tight approximation algorithms for $p$-mean welfare under subadditive valuations. arXiv preprint arXiv:2005.07370, 2020.

[10] Steven J Brams and Alan D Taylor. Fair Division: From cake-cutting to dispute resolution. Cambridge University Press, 1996.

[11] Felix Brandt, Vincent Conitzer, Ulle Endriss, Jérôme Lang, and Ariel D. Procaccia, editors. Handbook of Computational Social Choice. Cambridge University Press, 2016.

[12] Ioannis Caragiannis, Nick Gravin, and Xin Huang. Envy-freeness up to any item with high nash welfare: The virtue of donating items. In Proceedings of the 2019 ACM Conference on Economics and Computation (EC), pages 527-545. ACM, 2019.

[13] Ioannis Caragiannis, David Kurokawa, Hervé Moulin, Ariel D Procaccia, Nisarg Shah, and Junxing Wang. The unreasonable fairness of maximum Nash welfare. ACM Transactions on Economics and Computation (TEAC), 7(3):1-32, 2019.

[14] Suchan Chae and Hervé Moulin. Bargaining among groups: an axiomatic viewpoint. International Journal of Game Theory, 39(1-2):71-88, 2010.

[15] Bhaskar Ray Chaudhury, Yun Kuen Cheung, Jugal Garg, Naveen Garg, Martin Hoefer, and Kurt Mehlhorn. On fair division for indivisible items. In Proceedings of the 38th IARCS annual Conference on Foundations of Software Technology and Theoretical Computer Science (FSTTCS), pages 25:1-17. Springer, 2018.

[16] Bhaskar Ray Chaudhury, Jugal Garg, and Ruta Mehta. Fair and efficient allocations under subadditive valuations. arXiv preprint arXiv:2005.06511, 2020.

[17] Richard Cole and Vasilis Gkatzelis. Approximating the Nash social welfare with indivisible items. In Proceedings of the 47th ACM Symposium on Theory of Computing (STOC), pages 371-380. ACM, 2015. 
[18] Richard Cole, Nikhil Devanur, Vasilis Gkatzelis, Kamal Jain, Tung Mai, Vijay V Vazirani, and Sadra Yazdanbod. Convex program duality, Fisher markets, and Nash social welfare. In Proceedings of the 2017 ACM Conference on Economics and Computation (EC), pages 459-460. ACM, 2017.

[19] Sami Davies, Thomas Rothvoß, and Yihao Zhang. A tale of Santa Claus, hypergraphs and matroids. In Proceedings of the 31st Annual ACM-SIAM Symposium on Discrete Algorithms (SODA), pages 2748-2757. SIAM, 2020.

[20] Dagmawi Mulugeta Degefu, Weijun He, Liang Yuan, and Jian Hua Zhao. Water allocation in transboundary river basins under water scarcity: a cooperative bargaining approach. Water resources management, 30(12):4451-4466, 2016.

[21] Ran Duan, Jugal Garg, and Kurt Mehlhorn. An improved combinatorial polynomial algorithm for the linear Arrow-Debreu market. In Proceedings of the 27th Annual ACM-SIAM Symposium on Discrete Algorithms (SODA), pages 90-106. SIAM, 2016.

[22] Edmund Eisenberg. Aggregation of utility functions. Management Science, 7(4):337-350, 1961.

[23] Edmund Eisenberg and David Gale. Consensus of subjective probabilities: The pari-mutuel method. The Annals of Mathematical Statistics, 30(1):165-168, 1959.

[24] Uriel Feige. On maximizing welfare when utility functions are subadditive. SIAM Journal on Computing (SICOMP), 39(1):122-142, 2009.

[25] Satoru Fujishige and Zaifu Yang. A note on Kelso and Crawford's gross substitutes condition. Mathematics of Operations Research, 28(3):463-469, 2003.

[26] Jugal Garg and Peter McGlaughlin. Improving Nash social welfare approximations. J. Artif. Intell. Res., 68:225-245, 2020.

[27] Jugal Garg, Martin Hoefer, and Kurt Mehlhorn. Satiation in Fisher markets and approximation of Nash social welfare. arXiv preprint arXiv:1707.04428, 2017.

[28] Jugal Garg, Martin Hoefer, and Kurt Mehlhorn. Approximating the Nash social welfare with budget-additive valuations. In Proceedings of the 29th annual ACM-SIAM Symposium on Discrete Algorithms (SODA), pages 2326-2340. SIAM, 2018.

[29] Jugal Garg, Edin Husić, and László A. Végh. Auction algorithms for market equilibrium with weak gross substitute demands and their applications. arXiv preprint arXiv:1908.07948, 2019.

[30] Jugal Garg, Pooja Kulkarni, and Rucha Kulkarni. Approximating Nash social welfare under submodular valuations through (un)matchings. In Proceedings of the 2020 ACM-SIAM Symposium on Discrete Algorithms, SODA 2020, pages 2673-2687, 2020.

[31] Jonathan Goldman and Ariel D Procaccia. Spliddit: Unleashing fair division algorithms. ACM SIGecom Exchanges, 13(2):41-46, 2015.

[32] Martin Grötschel, László Lovász, and Alexander Schrijver. Geometric algorithms and combinatorial optimization, volume 2. Springer Science \& Business Media, 2012.

[33] Faruk Gul and Ennio Stacchetti. Walrasian equilibrium with gross substitutes. Journal of Economic theory, 87(1):95-124, 1999. 
[34] John C Harsanyi and Reinhard Selten. A generalized Nash solution for two-person bargaining games with incomplete information. Management science, 18(5-part-2):80-106, 1972.

[35] Harold Houba, Gerard van der Laan, and Yuyu Zeng. Asymmetric Nash solutions in the river sharing problem. 2013.

[36] Rishabh Iyer and Jeff Bilmes. Polyhedral aspects of submodularity, convexity and concavity. arXiv preprint arXiv:1506.07329, 2015.

[37] Kamal Jain. A polynomial time algorithm for computing an Arrow-Debreu market equilibrium for linear utilities. SIAM Journal on Computing (SICOMP), 37(1):303-318, 2007.

[38] Ehud Kalai. Nonsymmetric Nash solutions and replications of 2-person bargaining. International Journal of Game Theory, 6(3):129-133, 1977.

[39] Mamoru Kaneko and Kenjiro Nakamura. The Nash social welfare function. Econometrica: Journal of the Econometric Society, pages 423-435, 1979.

[40] Frank Kelly. Charging and rate control for elastic traffic. European transactions on Telecommunications, 8(1):33-37, 1997.

[41] Alexander S Kelso Jr and Vincent P Crawford. Job matching, coalition formation, and gross substitutes. Econometrica: Journal of the Econometric Society, pages 1483-1504, 1982.

[42] Subhash Khot and Ashok Kumar Ponnuswami. Approximation algorithms for the max-min allocation problem. In Approximation, Randomization, and Combinatorial Optimization. Algorithms and Techniques, pages 204-217. Springer, 2007.

[43] Subhash Khot, Richard J Lipton, Evangelos Markakis, and Aranyak Mehta. Inapproximability results for combinatorial auctions with submodular utility functions. Algorithmica, 52(1):3-18, 2008 .

[44] Annick Laruelle and Federico Valenciano. Bargaining in committees as an extension of Nash's bargaining theory. Journal of Economic Theory, 132(1):291-305, 2007.

[45] Benny Lehmann, Daniel Lehmann, and Noam Nisan. Combinatorial auctions with decreasing marginal utilities. Games Econ. Behav., 55(2):270-296, 2006.

[46] Renato Paes Leme. EC'18 Tutorial: "Gross Substitutes: Combinatorial Structure and Algorithms". https://www. youtube.com/watch?v=FJF0Py48wK4\&t=424s, 2018 (accessed April, 2020).

[47] Wenzheng Li and Jan Vondrák. Estimating the nash social welfare for coverage and other submodular valuations. In Proceedings of the Thirty-First Annual ACM-SIAM Symposium on Discrete Algorithms (SODA). SIAM, 2021. to appear.

[48] László Lovász. Matroid matching and some applications. Journal of Combinatorial Theory, Series B, 28(2):208-236, 1980.

[49] Hervé Moulin. Fair division and collective welfare. MIT press, 2004.

[50] Kazuo Murota. Discrete convex analysis, volume 10 of SIAM monographs on discrete mathematics and applications. SIAM, 2003. 
[51] Kazuo Murota. Lecture "Extensions and Ramifications of Discrete Convexity Concepts". https://www . youtube.com/watch?v=E-WjIrVm5Yk\&t=8s, 2015 (accessed February, 2020).

[52] Kazuo Murota. Problems for "Discrete Convex Analysis". https://www.him.uni-bonn.de/ uploads/media/HIMSummerSchool15MurotaProblem.pdf, 2015 (accessed February, 2020).

[53] Kazuo Murota. Discrete convex analysis: A tool for economics and game theory. Journal of Mechanism and Institution Design, 1(1):151-273, 2016.

[54] John F Nash. The bargaining problem. Econometrica: Journal of the econometric society, pages $155-162,1950$.

[55] Yurii Nesterov and Vladimir Shikhman. Computation of Fisher-Gale equilibrium by auction. Journal of the Operations Research Society of China, 6(3):349-389, 2018.

[56] Nhan-Tam Nguyen, Trung Thanh Nguyen, Magnus Roos, and Jörg Rothe. Computational complexity and approximability of social welfare optimization in multiagent resource allocation. Autonomous agents and multi-agent systems, 28(2):256-289, 2014.

[57] Noam Nisan, Tim Roughgarden, Éva Tardos, and Vijay V Vazirani. Algorithmic game theory. Cambridge University Press, 2007.

[58] James B Orlin. Improved algorithms for computing Fisher's market clearing prices: Computing Fisher's market clearing prices. In Proceedings of the 42nd ACM Symposium on Theory of Computing (STOC), pages 291-300. ACM, 2010.

[59] Michael Ostrovsky and Renato Paes Leme. Gross substitutes and endowed assignment valuations. Theoretical Economics, 10(3):853-865, 2015.

[60] Renato Paes Leme. Gross substitutability: An algorithmic survey. Games and Economic Behavior, 106:294-316, 2017.

[61] Richard Rado. A theorem on independence relations. The Quarterly Journal of Mathematics, (1):83-89, 1942.

[62] Jack Robertson and William Webb. Cake-cutting algorithms: Be fair if you can. CRC Press, 1998.

[63] Jörg Rothe, editor. Economics and Computation, An Introduction to Algorithmic Game Theory, Computational Social Choice, and Fair Division. Springer, 2016.

[64] Andrzej Ruszczynski. Nonlinear optimization. Princeton University Press, 2011.

[65] Alexander Schrijver. Combinatorial optimization: Polyhedra and efficiency, volume 24. Springer Science \& Business Media, 2003.

[66] Lloyd S Shapley. Complements and substitutes in the optimal assignment problem. Naval Research Logistics Quarterly, 9(1):45-48, 1962.

[67] Ngoc Mai Tran. The finite matroid-based valuation conjecture is false. arXiv preprint arXiv:1905.02287, 2019.

[68] Hal R Varian. Equity, envy, and efficiency. Journal of Economic Theory, 9(1):63-91, 1974. 
[69] Vijay V Vazirani. The notion of a rational convex program, and an algorithm for the ArrowDebreu Nash bargaining game. Journal of the ACM (JACM), 59(2):1-36, 2012.

[70] László A Végh. A strongly polynomial algorithm for a class of minimum-cost flow problems with separable convex objectives. SIAM Journal on Computing (SICOMP), 45(5):1729-1761, 2016.

[71] Jan Vondrák. Optimal approximation for the submodular welfare problem in the value oracle model. In Proceedings of the 40th ACM Symposium on Theory of Computing (STOC), pages 67-74, 2008.

[72] H Peyton Young. Equity: in theory and practice. Princeton University Press, 1995.

[73] S Yu, EC van Ierland, H-P Weikard, and X Zhu. Nash bargaining solutions for international climate agreements under different sets of bargaining weights. International Environmental Agreements: Politics, Law and Economics, 17(5):709-729, 2017. 